\title{
Observation of the onset of torsion-induced, mode-specific dissipative intramolecular vibrational redistribution (IVR)
}

David J. Kemp, William D. Tuttle, Adrian M. Gardner, ${ }^{a}$ Laura E. Whalley, and Timothy G. Wright ${ }^{\mathrm{b}}$

School of Chemistry, University of Nottingham, University Park, Nottingham NG7 2RD, UK

a Present Address: Stephenson Institute for Renewable Energy, University of Liverpool, L69 7ZF, UK

bTim.Wright@nottingham.ac.uk

\section{Abstract}

Evidence is found showing that coupling with vibration-torsion ("vibtor") levels of both in-plane and out-of-plane vibrations is instrumental in causing dissipative intramolecular vibrational redistribution (IVR). Both zero-electron-kinetic-energy (ZEKE) and two-dimensional laser-induced fluorescence (2DLIF) spectroscopy are employed to investigate a series of bands located $\sim 1200 \mathrm{~cm}^{-1}$ above the $S_{1} \leftarrow S_{0}$ origin in $p$-fluorotoluene ( $p \mathrm{FT})$. Transitions in this wavenumber region have been the focus of a number of studies probing intramolecular vibrational redistribution (IVR). By recording both ZEKE and 2D-LIF spectra, a prepared $S_{1}$ population is projected onto both the ground state cation and ground state neutral energy states, respectively, giving added confidence to the assignments. The spectral region under discussion is dominated by a pair of fundamental bands, but for the first time, we present explicit evidence that this is complicated by contributions from a number of overtones and combinations, including vibtor levels. We deduce that very different extents of coupling are present across a $60 \mathrm{~cm}^{-1}$ window of the spectrum, even though the density of states (DOS) is similar; in particular, one of the fundamentals couples efficiently to the increasing bath of levels, while one does not. We explain this by the influence of serendipitous near-coincidences of same-symmetry levels. 


\section{Introduction}

The ability to describe the making and breaking of chemical bonds and the flow of energy through a molecule necessitates a knowledge of the internal energy level structure; in particular, vibrations and torsions. Building on work by Parmenter and coworkers, ${ }^{1,2,3,4,5,6,7}$ and the group of Weisshaar, ${ }^{8,9,10}$ recent work by the Lawrance group and ourselves has identified that vibration-torsional ("vibtor") coupling is of key importance in the following para-substituted molecules that contain methyl groups: toluene, ${ }^{11,12,13,14,15,16,17} \mathrm{pFT}^{18,19,20,21,22,23,24,25,26,27}$ and para-xylene $(p \mathrm{Xyl}) .^{25,28,29}$ Some of the most recent work has employed the technique of two-dimensional laser-induced fluorescence (2D-LIF), which has been reviewed recently. ${ }^{30}$

Following on from earlier work on $\mathrm{pFT}^{31,32}$, Seliskar et al. ${ }^{33}$ and Okuyama et al. ${ }^{34}$ presented laserinduced fluorescence (LIF) spectra under jet-cooled conditions, giving assignments of some of the vibrational bands. A number of the low-wavenumber bands have been reassigned to vibration-torsion (vibtor) levels by Zhao, ${ }^{5}$ which was confirmed in recent work by our group ${ }^{21}$ and that of Lawrance et al. ${ }^{22}$ An overview of the lowest $1250 \mathrm{~cm}^{-1}$ of the $S_{1} \leftarrow S_{0}$ REMPI spectrum is shown in Figure 1. Recently, we have examined the bands close to $400 \mathrm{~cm}^{-1}$ (Ref. 26) and those at $800 \mathrm{~cm}^{-1}$ (Ref. 27) using 2D-LIF, and compared the results to our earlier ZEKE work. ${ }^{18,21,23}$ The assignments of the 2D-LIF spectra indicated that both vibrational interactions and vibration-torsional coupling were occurring.

In the present work, we examine the $1200 \mathrm{~cm}^{-1}$ region of the spectrum - see Figure 1 . This region of the spectrum contains two strong fundamental transitions, and these have been assigned by Okuyama et al. ${ }^{34}$ with LIF, and confirmed by our later ZEKE study. ${ }^{18}$ What was remarkable about the ZEKE spectra was that the one recorded from the lower fundamental $\left(1196 \mathrm{~cm}^{-1}\right)$ was highly structured, with very limited broadness in the baseline, while the one recorded via the higher one $\left(1232 \mathrm{~cm}^{-1}\right)$ had a very large amount of complicated overlapped structure across a wide range. This was interpreted as a significant increase in IVR in the latter, despite its being only $36 \mathrm{~cm}^{-1}$ higher in wavenumber. These spectra were further discussed in Refs. 19 and 20 in tandem with additional time-resolved data from the Reid group at Nottingham. In particular, in was noted therein that there has been some ambiguity in the labelling of vibrational levels previously studied, and effects of different experimental conditions were discussed; $;^{19}$ also, comparisons between observations for toluene, toluene- $d_{3}$ and $p \mathrm{FT}$ were made. ${ }^{20}$ Finally, more-recent work from the Reid group ${ }^{35}$ has looked at the same fundamentals, although the focus of that work was on combination levels involving these, located higher in wavenumber. 
Both a combination of an increased density of states (DOS) and symmetry-allowed vibtor coupling have been invoked to rationalize the rapid increase in interactions that occur in such molecules and which drive energy dispersal. ${ }^{25}$ In the present work, we consider a $\sim 6 \mathrm{~cm}^{-1}$ region close to $1200 \mathrm{~cm}^{-1}$ internal energy of the $\mathrm{S}_{1}$ state of $p \mathrm{FT}$. Very different intramolecular vibrational redistribution (IVR) behaviour has been observed for two fundamental vibrations located here. It has been hypothesised that the presence of a methyl group is responsible, but over such a narrow range the DOS is not expected to change enormously, and so the reason for the difference is unclear. Here, we examine both the abovementioned fundamental levels and other levels that are located in the $1190-1250 \mathrm{~cm}^{-1}$ range. In this region we expect to see combinations involving levels on which we have recently reported, at $\sim 400 \mathrm{~cm}^{-1}$ (Ref. 26) and $\sim 800 \mathrm{~cm}^{-1}$ (Ref. 27); additionally, combinations involving the levels at $\sim 400 \mathrm{~cm}^{-1}$ and those at $\sim 845 \mathrm{~cm}^{-1}$ (Ref. 24 ) may be expected to lie towards the higher wavenumber end of this region - see Figure 1. We piece together evidence from the 2D-LIF and ZEKE spectra presented in the present work to assign this region more completely than has been done before. We conclude that one of the fundamentals is able to couple efficiently to the increasing number of levels, via interactions with vibtor levels, while the other is not. We discuss the rationale for this.

\section{Experimental}

The 2D-LIF apparatus is the same as that employed recently. ${ }^{24}$ The vapour above room temperature para-fluorotoluene (99\% purity, Alfa Aesar) was seeded in $\sim 5$ bar of Ar and the gaseous mixture passed through a General Valve pulsed nozzle $(750 \mu \mathrm{m}, 10 \mathrm{~Hz}$, opening time of 180-210 $\mu \mathrm{s})$ to create a free jet expansion. This was intersected at $X / D \sim 20$ by the frequency-doubled output of a single dye laser (Sirah CobraStretch), operating with C540A. The fluorescence was collected, collimated and focused onto the entrance slits of a $1.5 \mathrm{~m}$ Czerny Turner spectrometer (Sciencetech 9150) operating in singlepass mode, dispersed by a 3600 groove/mm grating, and $\sim 300 \mathrm{~cm}^{-1}$ windows of the dispersed fluorescence collected by a CCD camera (Andor iStar DH334T). At a fixed grating angle of the spectrometer, the excitation laser was scanned, and at each excitation wavenumber the camera image was accumulated for 2000 laser shots. This allowed a plot to be produced of fluorescence intensity versus both the excitation laser wavenumber and the wavenumber of the emitted and dispersed fluorescence, termed a 2D-LIF spectrum. ${ }^{30}$

We have also recorded some separate dispersed fluorescence (DF) spectra with higher averaging to get better signal-to-noise than simply taking a vertical slice through the 2D-LIF image. These DF spectra 
were recorded with the same spectrometer as for the 2D-LIF spectra, and were recorded three times accumulating over 5000 shots each time, and an average taken of these.

The REMPI/ZEKE apparatus was the same as that used in earlier work. ${ }^{18}$ The focused, frequencydoubled outputs of the two dye lasers (Sirah CobraStretch) were overlapped spatially and temporally and passed through a vacuum chamber coaxially and counterpropagating, where they intersected a free jet expansion of $p \mathrm{FT}$ in Ar between two biased electrical grids located in the extraction region of a time-of-flight mass spectrometer, which was employed in the REMPI experiments. These grids were also used in the ZEKE experiments by application of pulsed voltages, giving typical fields $(F)$ of $\sim 10 \mathrm{~V}$ $\mathrm{cm}^{-1}$, after a delay of up to $2 \mu \mathrm{s}$, where this delay was minimized while avoiding the introduction of excess noise from the prompt electron signal. The resulting ZEKE bands had widths of $\sim 5-7 \mathrm{~cm}^{-1}$. Electron and ion signals were recorded on separate sets of microchannel plates.

The excitation laser operated with C503 and was pumped with the third harmonic ( $355 \mathrm{~nm}$ ) of a Surelite III Nd:YAG laser. The ionization laser operated with Pyromethene 597, pumped with the second harmonic $(532 \mathrm{~nm}$ ) of a Surelite I Nd:YAG laser. The fundamental outputs produced by each dye laser were frequency doubled.

\section{Results and assignments}

\subsection{Nomenclature and labelling}

\subsubsection{Vibrational and Torsional Labelling}

Since neither Wilson ${ }^{36} /$ Varsányi $^{37}$ nor Mulliken ${ }^{38} /$ Herzberg $^{39}$ notations are appropriate for the vibrations of $p \mathrm{FT}^{40,41}$ we shall employ the $D_{i}$ labels from Ref. 41 . In other papers, we have provided correlations with the labels used in previous work to aid the reader in referring to earlier studies. ${ }^{23,26}$ Note that we shall refer to previously calculated values (B3LYP/aug-cc-pVTZ) of the vibrational wavenumbers in the three electronic states pertinent to the present study, presented in our previous work - those for the $S_{1}$ and $D_{0}{ }^{+}$states are taken from Ref. 23, while those for the $S_{0}$ state are from Ref. 41. 
Although referred to in terms of Wilson nomenclature in earlier work, in fact a detailed analysis of the vibrational motions of various symmetric and asymmetric disubstituted benzenes ${ }^{41}$ showed that the $D_{5}$ and $D_{6}$ vibrations were in-phase and out-of-phase motions of the $C-X$ stretches, where $\mathrm{X}$ is the substituent in the symmetrically-substituted molecules. In the asymmetrically-substituted analogues, these vibrations evolve into localized stretches: in the halotoluenes, $D_{5}$ was assigned as the $C-\mathrm{Hal}$ stretch, while $D_{6}$ was identified as the $\mathrm{C}-\mathrm{CH}_{3}$ stretch, where Hal represents a halogen atom. ${ }^{41}$

Since the $G_{12}$ molecular symmetry group (MSG) is appropriate for vibtor levels in $p F T$, we shall use these symmetry labels throughout. In addition, torsional levels will be labelled via their $m$ quantum number. (The reader may find it useful to refer to previous work ${ }^{15,16,17,21,28}$ if they are not familiar with these labels.) The correspondence between the $C_{2 v}$ point group labels and the $G_{12}$ MSG ones are given in Table I. To calculate the overall symmetry of a vibtor level, it is necessary to use the corresponding $G_{12}$ label for the vibration, and then find the direct product with the symmetry of the torsion (Table I), noting that a $D_{3 h}$ point group direct product table can be used, since the $G_{12}$ MSG and the $D_{3 h}$ point group are isomorphic.

Under the free-jet expansion conditions employed here, almost all of the molecules are expected to be cooled to their zero-point vibrational level and thus essentially all $S_{1} \leftarrow S_{0}$ pure vibrational excitations are expected to be from this level. In contrast, owing to nuclear-spin and rotational symmetry, the molecules can be in one of the $m=0$ or $m=1$ torsional levels. ${ }^{28,42}$

\subsubsection{Coupling and transitions}

If an anharmonic vibration is close in wavenumber to one or more combination or overtone vibrational levels that has the same overall symmetry, then "off-diagonal" anharmonic interactions can occur, with the simplest example of two interacting states being the classic Fermi resonance (FR). ${ }^{43}$ The noninteracting levels are termed zero-order states (ZOSs), and their interaction leads to the formation of eigenstates that are linear combinations of these, and will be at different wavenumbers to the original ZOSs. ${ }^{39}$ For molecules that contain a hindered internal rotor, and if vibration-torsional coupling occurs, then the ZOSs can also be torsional or "vibtor" levels. The end result of such interactions is the formation of eigenstates which facilitate delocalization of energy through widespread motion of the molecule. Such couplings are only expected to be significant for small changes, $\Delta v \leq 3$, of the vibrational quantum number, and also for changes, $\Delta m$, of $0, \pm 3$ or \pm 6 in the torsional quantum number in descending order of likely strength. ${ }^{16,21,28,44,45}$ 
In electronic spectroscopy, if we assume a non-coupled picture initially, then a vibrational, torsional or vibtor ZOS can be bright (i.e. it has a significant transition intensity) or dark (i.e. it has no, or a very small transition intensity); these are often termed a zero-order bright (ZOB) state and a zero-order dark (ZOD) state, respectively. Following interaction, the resulting eigenstates will be composed of mixtures of $Z O B$ and $Z O D$ state character and so more transitions will become observable in the spectrum as a result of the interaction, by virtue of the $\mathrm{ZOB}$ character.

When designating excitations, we shall generally omit the lower level, since it will be obvious from the jet-cooled conditions; similarly, for emissions, we shall omit the upper level, as that will be obvious from the excitation and context. In the usual way, vibrational transitions will be indicated by the cardinal number, $i$, of the $D_{i}$ vibration, followed by a super/subscript specifying the number of quanta in the upper/lower states, respectively; torsional transitions will be indicated by $m$ followed by its value. Finally, vibtor transitions will be indicated by a combination of the vibrational and torsional transition labels. E.g. $5^{1} \mathrm{~m}^{0}$ implies an excitation from the torsionless (implied by symmetry) zero-point vibrational level in the $S_{0}$ state to the $S_{1}$ level with one quantum, of $D_{5}$ excited, and no torsional excitation, while $5^{1} m^{1}$ implies an excitation from the zero-point vibrational level, but with one quantum of torsion (implied by symmetry), in the $S_{0}$ state to the $S_{1}$ level with one quantum, of $D_{5}$ excited, and one quantum of torsion. If no $m$ values are specified, then the transition label refers to transitions involving both $m=0$ and $m=1$, whose transition wavenumbers are expected to be coincident at the present resolution.

The wavenumbers of the levels will be given with respect to the relevant zero-point level in each electronic state, but noting that some excitations will originate from the $m=1$ level in $S_{0}$ and those transition energies are given with respect to that level, as usual. The $\mathrm{S}_{1} \leftrightarrow \mathrm{S}_{0}$ origin is located at $36860.0 \mathrm{~cm}^{-1}$ (Ref. 22). The most intense transition is generally expected to be that for which no change in the vibrational, or both vibrational and torsional, quantum numbers occurs; these will be designated as $\Delta v=0$ or $\Delta(v, m)=0$ transitions. As has become common usage, we will generally refer to a level using the notation of a transition, with the level indicated by the specified quantum numbers, with superscripts indicating levels in the $S_{1}$ state and subscripts indicating levels in the $S_{0}$ state; since we will also be referring to levels in the ground state cation, $\mathrm{D}_{0}{ }^{+}$, we shall indicate those levels with superscripts with a preceding superscripted + sign. Also, the eigenstates will often be referred to by the dominant contribution from one of the ZOSs, with the context implying if an admixture of other ZOSs is present. 2D-LIF band positions will be indicated by a pair of (excitation, emission) wavenumbers, and the corresponding transitions similarly. 


\subsection{Overall comments on the spectra}

\subsubsection{The $S_{1} \leftarrow S_{0}$ spectrum}

In Figure 1, we show a REMPI spectrum of $p F T$ up to $1250 \mathrm{~cm}^{-1}$. Indicated are a set of bands at $\sim 400$ $\mathrm{cm}^{-1}$ that are dominated by $14^{2}, 29^{1}$ and $11^{1}$ transitions,,$^{18,21,22,26}$ but where there are also additional, including vibtor, transitions. ${ }^{21,22,26} \mathrm{At} \sim 800 \mathrm{~cm}^{-1}$ there are a further set of transitions, dominated by a pair of levels that largely comprise a FR between the $9^{1}$ and $29^{2}$ levels, ${ }^{23,27,46,47}$ but again, there are other transitions in this region, including those involving vibtor levels (see Refs. 23 and 27). At 845 $\mathrm{cm}^{-1}$ there is the $18^{2}$ transition, which has been shown to be interacting strongly with several vibtor levels at this wavenumber (see Ref. 24), while at $\sim 1015 \mathrm{~cm}^{-1}$ there is a band that is the subject of ongoing work. At $\sim 1200 \mathrm{~cm}^{-1}$, there are a series of bands which are dominated by two fundamental transitions, assigned as $6^{1}$ and $5^{1}$; in earlier work, these have mainly been referred to with Wilson labels $v_{13}$ and $v_{7}$, respectively. ${ }^{18,20,34}$ It is clear, however, that further bands are present and expanded views of this wavenumber region are presented in Figure 2, recorded using three different techniques: REMPI, LIF and integration of the 2D-LIF spectrum (see Section 3.2.3). Immediately apparent is the relative similarity of the LIF and integrated 2D-LIF spectra. However, the relative intensities of the two most intense bands is clearly different to those in the REMPI spectrum, and indeed the higherwavenumber feature has an even greater relative intensity in some REMPI scans. ${ }^{18}$ This is supportive of there being some time-dependent effect on the $5^{1}$ band, with REMPI being a far faster technique than LIF, and higher laser power densities favouring ionization over $\mathrm{S}_{1}$ population-loss mechanisms.

We shall now present the ZEKE spectra and the 2D-LIF spectrum, each recorded across the spectral region shown in Figure 2 . We shall then discuss these together in deducing the detailed assignments.

\subsubsection{ZEKE Spectra}

In Figure 3, we show ZEKE spectra recorded at each of the indicated positions; the ZEKE spectrum recorded via the origin is shown for comparison purposes. The indicated transitions arise from the overall assignment to be discussed in Section 3.3. We highlight that the ZEKE spectrum via the origin has a flat baseline throughout. The spectrum recorded at $1196 \mathrm{~cm}^{-1}$ has a largely flat baseline until $\sim 1000 \mathrm{~cm}^{-1}$, and then a small amount of underlying complicated, overlapped structure is seen thereafter. The strong ZEKE bands in this spectrum can be assigned as ${ }^{+} 6^{1},{ }^{+} 6^{1} 11^{1}$ and ${ }^{+} 6^{1} 9^{1}$. All other 
spectra have significant contributions from a wide range of underlying overlapped structure, although some caution is required for ZEKE spectra that were recorded via levels corresponding to weak bands in the REMPI spectrum, where the background can be relatively significant. We highlight the spectrum recorded via the level corresponding to the intense band at $1232 \mathrm{~cm}^{-1}$, which shows this broad structure, but there are clear bands seen on top of this, notably those assigned to ${ }^{+} 5^{1},{ }^{+} 5^{1} 11^{1}$ and ${ }^{+} 5^{1} 9^{1}$. The ZEKE spectra recorded at $1196 \mathrm{~cm}^{-1}$ and $1232 \mathrm{~cm}^{-1}$ are very similar to those reported and discussed previously. ${ }^{18,19,20}$ Other bands and indicated assignments will be discussed in Section 3.3.

\subsubsection{D-LIF spectrum}

In Figure 4, we show a composite overview of the 2D-LIF spectra recorded across the excitation range corresponding to that of Figure 2, and covering emissions to the origin up until $2550 \mathrm{~cm}^{-1}$ of the $\mathrm{S}_{0}$ state, selected to cover the $\Delta v=0$ regions of transitions that might be contributing to the spectrum in Figure 2. Integrating the 2D-LIF spectrum vertically gives an excitation spectrum similar to the REMPI and LIF spectra - see Figure 2. It is evident that there are two main vertical "stripes" of activity located around the $1196 \mathrm{~cm}^{-1}$ and $1232 \mathrm{~cm}^{-1}$ excitations, i.e. as a result of exciting $6^{1}$ and $5^{1}$, respectively. It is also clear that there is a significant amount of Franck-Condon (FC) emission activity as a result of these two excitations, and that there is more intensity associated with the $6^{1}$ excitation than with the $5^{1}$ excitation. In Figure 5 we shown DF spectra recorded through the centres of the $6^{1}$ and $5^{1}$ excitations and, for comparative purposes, also through the origin. Expanded relevant sections of the 2D-LIF spectra in Figure 2 are given in Figure 6. From Figure 5, it is clear that there is much more broad underlying emission structure when exciting $5^{1}$ than there is for $6^{1}$, consistent with the behaviour seen in the ZEKE spectra (see Figure 3). As indicated by the additional structure seen in Figures 2 and 4, there is additional activity at other excitation wavenumbers, located both between $6^{1}$ and $5^{1}$, as well as to higher wavenumber than $5^{1}$, and this will be discussed and assigned in tandem with the ZEKE spectra in the following subsection.

\subsection{Assignments}

We commence by noting that the previous ${ }^{18,19,20}$ and present ZEKE spectra (Figure 3 ) are consistent with the main two bands in Figure 2 being $6^{1}$ and $5^{1}$. The 2D-LIF spectrum is also consistent with this, with outline assignments indicated in the DF spectra in Figure 5 (further assignments are given in Figure 6). Thus, the $\left(6^{1}, 6_{1}\right)$ and $\left(5^{1}, 5_{1}\right) \Delta v=0$ bands are located at $(1196,1215) \mathrm{cm}^{-1}$ and $(1232,1241)$ $\mathrm{cm}^{-1}$, respectively. A series of other intense Franck-Condon $(\mathrm{FC})$ active combination and overtone 
bands involving the main transition can be seen to higher wavenumber in each case. As well as $11_{1}$ (see Figure 5), there are also other emissions, with those in the range $\sim 840-855 \mathrm{~cm}^{-1}$ being assigned as $14_{2}, 9_{1}$ and $29_{2}$. (Note that there was some indication that, as well as $9^{1}$ and $29^{2}$ being in Fermi resonance in the $S_{1}$ state, ${ }^{27,47}$ the $9_{1}$ and $29_{2}$ levels may be in Fermi resonance in $S_{0}$, and the same was true of ${ }^{+} 9^{1}$ and ${ }^{+} 29^{2}$ in $\mathrm{D}_{0}{ }^{+} \cdot{ }^{27}$ )

We now consider the combination bands involving one of the $S_{1}$ levels previously discussed ${ }^{24,26,27}$ at $\sim 400 \mathrm{~cm}^{-1}$ and another located at $\sim 800 \mathrm{~cm}^{-1}$ or $\sim 845 \mathrm{~cm}^{-1}$ (see Figure 1) that are expected to lie in the 1190-1250 $\mathrm{cm}^{-1}$ range. To aid the reader, in Figure 7 we show the expected positions of the various combinations, indicated by shifting the region of the spectrum at $\sim 400 \mathrm{~cm}^{-1}$ to positions that indicate where the various combinations are expected. If these combinations have significant intensity, we should be able to identify activity from them in the 2D-LIF and ZEKE spectra, although we need to consider that interactions may shift levels from the expected position. In addition, it is possible for additional ZOB states to contribute to this region of the spectrum, analogous to some of those seen at similar wavenumbers in our recent studies of $p$-difluorobenzene $(p D F B)^{48}$ and $p$ chlorofluorobenzene ( $p$ CIFB), ${ }^{49,50}$ but noting that the higher symmetry of $p$ DFB means that some transitions are symmetry forbidden, notably $6^{1}$. Between different molecules, however, the brightness of any transition can vary, as can its wavenumber, with the latter potentially causing levels to move, affecting the possible interactions. Furthermore, the presence of the methyl group in pFT leads to vibtor levels built on each vibrational level, dramatically increasing the possibilities for coupling - see the discussion in Ref. 25 concerning comparison between the abovementioned three molecules and also $p$ Xyl.

\subsubsection{Combinations of $\left(14^{2}, 29^{1}\right.$ and $\left.11^{1}\right)$ with $\left(9^{1}\right.$ and $\left.29^{2}\right)$}

We first consider the levels expected to arise from the two $9^{1} / 29^{2} \mathrm{FR}$ levels (located at $~ 800 \mathrm{~cm}^{-1}$ ) in combination with the three main $400 \mathrm{~cm}^{-1}$ transitions, $14^{2}, 29^{1}$ and $11^{1}-$ see Figures 1 and 7.

Although there is an emission to $9_{1} 14_{2}\left(1673 \mathrm{~cm}^{-1}\right)$ - see Figure 6 - this is particularly intense via $6^{1}$. One explanation for this would be FC activity, but it could also indicate that the $9^{1} 14^{2}$ level is coincident with $6^{1}$. Since this coincidence would imply there was very little interaction between these two levels, and combined with the fact that we see no clear ${ }^{+} 9^{1} 14^{2}$ band in the ZEKE spectrum when exciting via $6^{1}$, we favour FC activity. 
In Figure 8 , it can be seen that, as well as the clear FC $\left(6^{1}, 14_{2}\right)$ emission, there is also a secondary $14_{2}$ emission when exciting at $1198 \mathrm{~cm}^{-1}$ (see also the integrated trace in the lower half of this figure), and we suggest that this could be associated with the $9^{1} 14^{2} \Delta v=-1$ transition, with there being a ZEKE band at the correct position (Figure 3 ), as well as a clear ${ }^{+} 14^{2}$ band when exciting at this wavenumber. (Although $14^{6}$ and $14^{4} 29^{1}$ are expected close to this excitation wavenumber neither ${ }^{+} 14^{6}$ nor ${ }^{+} 14^{4} 29^{1}$ bands are clearly evident, although there is a ${ }^{+} 14^{4}$ band at $1403 \mathrm{~cm}^{-1}$; in addition, the corresponding emission bands are not definitively seen in the 2D-LIF spectrum, and so evidence for activity involving these levels is weak.) We also cannot rule out a possible contribution to the $\sim 1530 \mathrm{~cm}^{-1}$ ZEKE band from ${ }^{+} 14^{2} 29^{2}$, which would also be consistent with FC activity of ${ }^{+} 14^{2}$ band. Although there is no definitive $14_{2} 29_{2}$ emission band seen, the $14_{2}$ band could also be associated with emission from $14^{2} 29^{2}$. In summary, although there is some evidence for $9^{1} 14^{2}$ and $14^{2} 29^{2}$ (and possibly $14^{4} 29^{1}$ and $14^{6}$ ) activity when exciting at $1198 \mathrm{~cm}^{-1}$ it is not wholly conclusive.

We now move on to $9^{1} 29^{1}$ and $29^{3}$, recalling that these are more correctly thought of as combinations of $29^{1}$ with each of the $\left(9^{1} \ldots 29^{2}\right)$ and $\left(29^{2} \ldots 9^{1}\right)$ FR pair. The clearest evidence for this activity is from the $29_{1}$ emission, which shows two significant areas of activity at excitations of $1193 \mathrm{~cm}^{-1}$ and 1203 $\mathrm{cm}^{-1}$ (see Figure 8). These regions of activity are identified as the $\left(29^{3}, 29_{3}\right) \Delta v=0$ band at $(1203,1275)$ $\mathrm{cm}^{-1}$, with the band at $(1193,1264) \mathrm{cm}^{-1}$ being $\left(9^{1} 29^{1}, 9_{1} 29_{1}\right)$; further, there is some evidence for cross activity between these bands, consistent with FR between the $9^{1} 29^{1}$ and $29^{3}$ levels, as there was 27,47 between the $9^{1}$ and $29^{2}$ levels. In both cases the $29_{3}$ emission is the strongest, consistent with the $29_{2}$ band being the stronger when exciting via both of the $9^{1} / 29^{2} \mathrm{FR}$ levels. ${ }^{27}$ The weak $\left(6^{1}, 29_{1}\right)$ activity at $(1196,424) \mathrm{cm}^{-1}$ is assigned as Herzberg-Teller (HT) activity associated with the $6^{1}$ transition, similar to that seen via the origin. ${ }^{21,22,26}$

We expect the combinations of $11^{1}$ with the $9^{1} / 29^{2} \mathrm{FR}$ pair at excitation wavenumbers of around 1205 $\mathrm{cm}^{-1}$ and $1211 \mathrm{~cm}^{-1}$. There is clear FC activity for both $9_{1} 11_{1}\left(1295 \mathrm{~cm}^{-1}\right)$ and $11_{1} 29_{2}\left(1302 \mathrm{~cm}^{-1}\right)$ following $6^{1}$ excitation, so that the emission wavenumber is secure. We thus assign the weak band at $(1204,1302) \mathrm{cm}^{-1}$ as $\left(9^{1} 11^{1}, 11_{1} 29_{2}\right)$, in line with the expected greater intensity of $11_{1} 29_{2}$ emission when exciting via both FR components, and the very weak $(1204,1296) \mathrm{cm}^{-1}$ band to $\left(9^{1} 11^{1}, 9_{1} 11_{1}\right)$. Surprisingly, we only see the faintest traces of the partner $\left(11^{1} 29^{2}, 11_{1} 29_{2}\right)$ band, expected at around $(1211,1302) \mathrm{cm}^{-1}$ and no clear evidence for $11^{1} 14^{4}$ activity was seen. The ZEKE activity (Figure 3 ) shows slightly more convincing evidence of the activity of both of these $S_{1}$ levels, although there was no ZEKE spectrum recorded following excitation at $1211 \mathrm{~cm}^{-1}$. 
Finally, a weak band at $(1198,1528) \mathrm{cm}^{-1}$ is a reasonably good match for $\left(11^{1} 15^{1} 20^{1}, 11_{1} 15_{1} 20_{1}\right)$, expected at $(1203,1524) \mathrm{cm}^{-1}$ - see Figure 6 ; however, it is not possible to identify the corresponding ${ }^{+} 11^{1} 15^{1} 20^{1}$ ZEKE band definitively, owing to a number of transitions being expected at this wavenumber in the spectrum - see Figure 3 ; on the other hand, the FC-allowed ${ }^{+} 15^{1} 20^{1}$ band is seen, supporting the activity of the $11^{1} 15^{1} 20^{1}$ level.

In summary, the main expected combinations between the $\sim 400 \mathrm{~cm}^{-1}$ and $800 \mathrm{~cm}^{-1}$ set of lines do seem to appear in this wavenumber region, and although they were sometimes weak in the 2D-LIF spectrum, they were often more apparent in the ZEKE spectrum. More clear in the 2D-LIF spectrum is the $9^{1} 29^{1} / 29^{3}$ FR interaction (Figure 8), while the separate bands are less easy to discern in the ZEKE spectra. Part of the explanation for these intensity variations may be different Franck-Condon factors (FCFs) for the ionization and emission processes.

\subsubsection{Combinations of $\left(14^{2}, 29^{1}\right.$ and $\left.11^{1}\right)$ with $\left(11^{2}\right.$ and $\left.12^{1} 14^{1}\right)$}

Higher in excitation wavenumber, at $\sim 1215 \mathrm{~cm}^{-1}$, we expect to find the combinations of the excitation bands that appear at $\sim 818 \mathrm{~cm}^{-1}, 12^{1} 14^{1}$ and $11^{2}$, with each of $29^{1}$ and $14^{2}$, at excitation wavenumbers of $\sim 1215 \mathrm{~cm}^{-1}$. However, no clear evidence of bands corresponding to these levels could be found.

Slightly higher than this, at $1226 \mathrm{~cm}^{-1}$, we would expect $11^{1} 12^{1} 14^{1}$ and $11^{3}$. We see an unexpectedly bright (compared to the corresponding FC-active band via $6^{1}$ ) emission at $(1230,1816) \mathrm{cm}^{-1}$, i.e. essentially coincident with excitation at $5^{1}$, which we assign to $11_{1} 12_{1} 14_{1}-$ see Figure 6 . Because of this unexpected brightness, we assign this band as the $\left(11^{1} 12^{1} 14^{1}, 11_{1} 12_{1} 14_{1}\right) \Delta v=0$ band, which is coincident, but apparently not interacting, with $5^{1}$. Note that in the cation, the expected wavenumber of ${ }^{+} 5^{1} 11^{1}\left(1772 \mathrm{~cm}^{-1}\right)$ is almost identical to ${ }^{+} 11^{1} 12^{1} 14^{1}\left(1775 \mathrm{~cm}^{-1}\right)$ and so both could be contributing to the ZEKE band seen at $1781 \mathrm{~cm}^{-1}$, when exciting at $1232 \mathrm{~cm}^{-1}$ - see Figure 3 . Weak $11_{3}$ activity is also seen when exciting close to $5^{1}$, but such activity is also seen via $6^{1}$, and so these may simply be attributed to FC activity.

\subsubsection{Other $\Delta v=0$ bands}

In Figure 9 we show another expanded view of the 2D-LIF spectrum where other $\Delta v=0$ bands are located. Quite an intense band at $(1208,1654) \mathrm{cm}^{-1}$ is assigned as $\left(14^{1} 16^{1} 29^{1}, 14_{1} 16_{1} 29_{1}\right)$, which is at the expected wavenumber in both $S_{0}$ and $S_{1}$; we would thus expect a ZEKE band at $1608 \mathrm{~cm}^{-1}$ when 
exciting at $1208 \mathrm{~cm}^{-1}$, and indeed a band is seen at $1607 \mathrm{~cm}^{-1}$ - see Figure 3 . We are comfortable with this assignment, since the wavenumbers of this combination are consistent across three electronic states and we observe that its three components are all active in the spectrum in different guises.

The band at $(1224,1639) \mathrm{cm}^{-1}$ is assigned as $\left(16^{2}, 162\right)$, and is in fairly good agreement with both the $S_{1}$ and $S_{0}$ values (unfortunately no ZEKE spectrum was recorded at this excitation wavenumber); the $16^{2}$ band was also active in $p$ DFB. ${ }^{48}$. A weak band at $(1226,1770) \mathrm{cm}^{-1}$ can be assigned as $\left(12^{1} 16^{1}\right.$, $\left.12_{1} 16_{1}\right)$.

The assignments above yield a wavenumber for $D_{16}$ in the $S_{1}$ state of $\sim 610 \mathrm{~cm}^{-1}$. Although this value is somewhat different to the calculated value ${ }^{23}$ of $651 \mathrm{~cm}^{-1}$, it is in excellent agreement with the experimentally-derived value of $609 \mathrm{~cm}^{-1}$, obtained from an assignment of the $16^{1} 20^{1}$ combination band. ${ }^{23}$ (Note that the tabulated experimental value of $607 \mathrm{~cm}^{-1}$ in Ref. 23 is incorrect.) This consistency of the wavenumber for $D_{16}$ in the $S_{1}$ state for the three abovementioned bands adds weight to this value, and suggests that an alternative, less favoured, value of $621 \mathrm{~cm}^{-1}$ that was discussed in Ref. 23 is incorrect.

To higher excitation wavenumber in Figure 9 , there is a band at $(1245,1900) \mathrm{cm}^{-1}$, which is consistent with being the $\left(12^{2}, 12_{2}\right)$ band; the $12^{2}$ band was also active in $p D F B .{ }^{48}$ We also see in the ZEKE spectrum in Figure 3, that when exciting at $1248 \mathrm{~cm}^{-1}$, a strong band was seen at $1977 \mathrm{~cm}^{-1}$ assignable to ${ }^{+} 12^{2}$. Interestingly, there is significant, overlapped underlying structure here, suggesting widespread coupling, which will be commented on in subsection 3.4. It is also interesting to see the ${ }^{+} 12^{1}$ band in this ZEKE spectrum, which is reminiscent of the activity of ${ }^{+} 14^{1}$ in other ZEKE spectra, despite being non-totally symmetric. Activity in such bands was discussed in relation to pDFB in Ref. 48.

We also saw very weak 2D-LIF bands (not shown), plausibly assignable as combinations of $18_{2}$ with $11_{1}$ and $29_{1}$, suggesting that the activity of the corresponding $S_{1}$ levels is low.

\subsubsection{Vibtor levels}

In examining the emission when exciting from $5^{1}$, a band is seen at $518 \mathrm{~cm}^{-1}$, which is the same band seen in our earlier study ${ }^{24}$ of the $S_{1}$ levels at $\sim 845 \mathrm{~cm}^{-1}$ and assigned to $18_{1} m_{2}$, i.e. a vibtor level of $e^{\prime \prime}$ symmetry - see Figure 10. This band was a signature that combinations of the $18^{1} \mathrm{~m}^{2}$ vibtor level were coupled to $18^{2} \mathrm{~m}^{1}$ in the $S_{1}$ state, also of $e^{\prime \prime}$ symmetry. In the present case, $9^{1} 18^{1} \mathrm{~m}^{2}$ would be expected at $\sim 1238 \mathrm{~cm}^{-1}$ and so very close to $5^{1}$; we thus conclude that $9^{1} 18^{1} \mathrm{~m}^{2}$ is coupled to $5^{1} \mathrm{~m}^{1}$, both being of 
$e^{\prime \prime}$ symmetry. There is a weak band that is tentatively assigned to $9_{1} 18_{1} m_{2}$ at around $1358 \mathrm{~cm}^{-1}$ (Figure 10), with the weakness being explained as being due to this level being a doorway state and so coupled to many other levels, explaining the rich emission coming from a range of different so-formed eigenstates: the $18_{1} m_{2}$ emission band is the resultant sum of FC-active emissions from all of these.

There is also clear weak background emission when exciting at $1246 \mathrm{~cm}^{-1}$, and some clue as to its origin comes from the observation of a 2D-LIF band at $(1246,476) \mathrm{cm}^{-1}$ - see Figure 10 . The emission can be assigned to $29_{1} m_{3(+)}$ and so it is then straightforward to deduce that the coupled level in the $\mathrm{S}_{1}$ state is likely to be $9^{1} 29^{1} \mathrm{~m}^{3(+)}$, which is expected to be close in wavenumber to $12^{2}$ and so may couple to its $m$ $=0$ level. Indeed, the extent of the $12_{2}$ emission band suggests coupling between these levels. A weak band at $(1245,1325) \mathrm{cm}^{-1}$ (not shown) is the likely $9^{1} 29^{1} \mathrm{~m}^{3(+)} \Delta(v, m)=0$ band.

In discussing the levels at $\sim 400 \mathrm{~cm}^{-1},{ }^{26}$ we identified $20^{2} \mathrm{~m}^{6(+)}$ vibtor activity, and the $9^{1} 20^{2} \mathrm{~m}^{6(+)}$ level would lie very close in energy to $6^{1}$; this would couple to only the $m=0$ level. Further evidence for this is provided by some weak emission bands observed when exciting at wavenumbers corresponding to $6^{1}: 204 \mathrm{~cm}^{-1}\left(m_{6(+)}\right), 350 \mathrm{~cm}^{-1}\left(20_{1} m_{6(+)}\right)$ and $492 \mathrm{~cm}^{-1}\left(20_{2} m_{6(+)}\right)-$ see Figure 10 . The $\Delta(v, m)=0$ band would be expected at around $1332 \mathrm{~cm}^{-1}$ and there are bands close to this value, and although it was not possible to associate one definitively with this transition, significant coupling would explain this being weak. We also see weak $29_{1} m_{3(+)}$ and $m_{3(+)}$ bands, which may be associated with another vibtor level coupling - a plausible candidate is $17^{1} 19^{1} 29^{1} \mathrm{~m}^{3(+)}$, which would appear at this wavenumber, and there are clear emissions close to $1505 \mathrm{~cm}^{-1}$, expected for the $\Delta(v, m)=0$ band (see Figure 10), but other levels, such as $6_{1} 20_{2}$ are also expected in this wavenumber region, and so it is not possible to associate a particular band with each transition. The $17^{1} 19^{1} 29^{1} \mathrm{~m}^{3(+)}$ level can only couple to the $6^{1} \mathrm{~m}^{0}$ level.

We also did look for activity associated with combination bands associated with emission or ionization from combination bands involving $14^{1} \mathrm{~m}^{6(-)}$, since this transition is observed at $\sim 400 \mathrm{~cm}^{-1}$ (Ref. 26), but could not unambiguously identify any such.

In the following section we shall discuss the above vibtor levels further, and their role in promoting IVR. 


\section{Discussion}

Referring to Figure 1 , in previous work on the $S_{1}$ state, evidence for Fermi resonances and coupling to vibtor levels has been found in the low wavenumber region $\left(<400 \mathrm{~cm}^{-1}\right), 21,22$ the bands at $\sim 400 \mathrm{~cm}^{-1}, 21$ the bands at $\sim 800 \mathrm{~cm}^{-1}, 23,47$ and the bands at $\sim 845 \mathrm{~cm}^{-1} .{ }^{24}$ However, it is the bands at $\sim 1200 \mathrm{~cm}^{-1}$, as studied in the present work, where evidence for widespread coupling is first apparent. ${ }^{18,19,20,35}$ The evidence from previous work is somewhat confusing, with uncertainties and apparent contradictions in the deductions. We thus briefly discuss that work, while rationalizing it in terms of the present data.

We first note that, as commented on in Refs. 19 and 20, some of the time-domain experiments by Parmenter's group ${ }^{1,2,6}$ have some inconsistencies as to the cited wavenumber employed in those studies, and also the designation of the vibration being excited. We also recall that $D_{6}$ is predominantly the $\mathrm{C}-\mathrm{CH}_{3}$ stretch, while $D_{5}$ is the C-F stretch. ${ }^{41}$ As such, with the large amplitude motion (the $\mathrm{CH}_{3}$ torsion) being the likely perpetrator of any acceleration, the expectation would be ${ }^{51,52}$ that the $D_{6}$ mode would be subject to more-widespread coupling than $D_{5}$, and this can clearly be seen not to be the case from the spectra and assignments discussed above. Furthermore, the ZEKE (see Figure 3), 2DLIF and DF (see Figures 4, 5 and 6) spectra show that there is only a very small amount of underlying broad structure when exciting $6^{1}$, while, in contrast, the time-resolved data in Refs. 20 and 35 indicate that there is significant loss of signal for this level, which would be consistent with widespread coupling. The data in Ref. 35 is in agreement that there is widespread coupling for $5^{1}$, and furthermore, it is deduced that the IVR lifetime for $5^{1}$ is about a quarter of that for $6^{1}$, consistent with the more significant underlying unresolved structure in the ZEKE, 2D-LIF and DF spectra. Moreover, the timedependent plots shown in Ref. 35 show only the faintest signs of quantum beating, and mostly are described by an exponential decay. Of particular interest is that the $6^{1}$ signal decays to about $50 \%$ of its original value by $100 \mathrm{ps}$, while the $5^{1}$ signal has reached about $10 \%$. The former observation is highlighted in Refs. 20 and 35, where it is suggested that only one of the $m$ levels efficiently facilitates vibtor coupling. No explicit comments are made in Ref. 35 regarding the remnant $5^{1}$ signal, but being less than $50 \%$ it would have to imply that both $m=0$ and $m=1$ levels undergo losses in intensity. The higher resolution of the ZEKE spectra in the present work, together with the added information from the 2D-LIF spectra on the nature of the $S_{1}$ vibrations and vibtor levels in the $\sim 1200 \mathrm{~cm}^{-1}$ region, provides more insight into the time-resolved observations.

First, we note that the ps laser used in the time-resolved slow-electron velocity-map imaging (SEVI) studies in Refs. 20 and 35 had a FWHM of $\sim 13 \mathrm{~cm}^{-1}$. Hence, when centred on the $6^{1}$ transition, this 
could be exciting a number of eigenstates, amongst which are $11^{1} 15^{1} 20^{1}, 9^{1} 29^{1}, 9^{1} 14^{2}, 29^{3}$, with other possibilities being $14^{6}, 14^{5} \mathrm{~m}^{6(-)}, 9^{1} 14^{1} \mathrm{~m}^{6(-)}$ and $14^{5} \mathrm{~m}^{6(-)}$; in addition, in the SEVI spectrum, unresolved from the main ${ }^{+} 6^{1}$ band are likely to be ${ }^{+} 9^{1} 29^{1},{ }^{+} 9^{1} 11^{1},{ }^{+} 29^{3}$ and a number of others. Thus, the timedependent SEVI signal that was monitored may well comprise a number of different contributions. That said, the fact that the intensity dropped to $50 \%$ after a few hundred ps, would be consistent with only one of the $m$ levels of the main $6^{1}$ transition undergoing widespread coupling. (Vibrationvibration anharmonic coupling to other totally-symmetric levels would occur for both $m=0$ and $m=$ 1 vibtor levels, and consequently would not explain the loss in intensity of just one of the $m$ levels.) Additionally, the growth in intensity of the $1370-1550 \mathrm{~cm}^{-1}$ region of the SEVI spectrum observed in Ref. 20 is consistent with a ZOB state coupling to a wide range of levels, and similar broad underlying structure is also evident in the ZEKE spectrum in Figure 3.

We have noted above that the $9^{1} 20^{2} m^{6(+)}$ and $17^{1} 19^{1} 29^{1} m^{3(+)}$ levels would lie very close in energy to $6^{1}$, and that these would couple to the $m=0$ level only. Also, once such vibration-torsional coupling has occurred, further interactions with other levels open up and this, together with couplings involving other ZOSs in this region, would explain the underlying structure seen in the ZEKE and DF spectra in Figures 3 and 5. That said, it does not seem that the ZEKE and 2D-LIF/DF spectra recorded when exciting $6^{1}$ are consistent with a $50 \%$ loss in intensity, suggesting that there may be another mechanism causing the time-dependent SEVI signa ${ }^{20}$ to decay. One such would be rotational dephasing ${ }^{53}$ occurring between the $6^{1} m^{0}$ rotational levels, caused by an $m$-and $J, K$-dependent vibtor interaction. ${ }^{27,47}$ Such dephasing in $p \mathrm{FT}$ was put forward with regards to the two main levels at $\sim 800$ $\mathrm{cm}^{-1}$ by Davies and Reid, ${ }^{47}$ and commented on further in our recent study of the same levels. ${ }^{27} \mathrm{As}$ noted above, the suggested vibtor interactions would be with just the $6^{1} m^{0}$ level, and so could cause the rotation-dependent coupling required for dephasing. If the dephasing were complete in a few 100 ps (as was in the case of the levels at $\sim 800 \mathrm{~cm}^{-1}$ ) 47 then this would be an explanation for the decay in the $6^{1}$ signal in Refs. 20 and 35. As discussed in Ref. 27, the rotational dephasing does not lead to a population change, and so in the frequency-resolved experiments no effect is seen from this; it only arises from the simultaneous excitation of the perturbed rotational levels. This explanation would then explain the very small unstructured background seen in the present work, and the very significant ( 50\%) drop in the " $6{ }^{1 "}$ SEVI signal. Given that the amount of unstructured background appears to be comparable in those SEVI spectra ${ }^{20,35}$ as in the present ZEKE and 2D-LIF/DF spectra, we conclude the two sets of results are consistent within this explanation. 
For the $5^{1}$ level, the situation is somewhat different, with only $10 \%$ of the ${ }^{+} 5^{1} \mathrm{SEVI}$ signal remaining after 100 ps. $^{35}$ We have seen that we observe the $18{ }_{1} m_{2}$ emission band when exciting at the wavenumber of $5^{1}$ (Figure 10) and from this we conclude that the $9^{1} 18^{1} \mathrm{~m}^{2}$ level is coupled to $5^{1} \mathrm{~m}^{1}$ in a similar way that $11^{1} 18^{1} \mathrm{~m}^{2}$ and $14^{2} 18^{1} \mathrm{~m}^{2}$ are coupled to $18^{2} \mathrm{~m}^{1}$ (Ref. 24). Assuming the coupling to $9^{1} 18^{1} \mathrm{~m}^{2}$ opens up efficient routes for second-order coupling of $5^{1} \mathrm{~m}^{1}$ to a significant set of bath states, then this could be a rationale for loss of $50 \%$ of the ${ }^{+} 5^{1}$ SEVI signal in Ref. 35 . Although there are a wealth of bands seen in the 2D-LIF/DF spectra at the wavenumber of $5^{1}$ (for example, see Figures 5, 6 and 10), it is difficult to assign them all uniquely, but there are various emission bands that can be assigned as analogous FC-active bands, but now in combination with $18_{1} m_{2}$, seen via $9^{1}$, but now active close to the $5^{1}$ position (Figure 10). In contrast to the ZEKE spectra in Ref. 24, we did not observe the ${ }^{+} 18^{1} m^{2}$ ZEKE band in the present work, but the UV intensity of the ionization laser was poor in the spectral region where this band is expected; for the same reason, other torsional levels were also not always seen in the ZEKE spectra that were seen here in emission. Overall, though, on the basis of earlier work and the 2D-LIF results, we are confident that the $5^{1} m^{1}$ level is strongly coupled to a background bath of states, with $9^{1} 18^{1} \mathrm{~m}^{2}$ acting as a doorway state. With this in mind, looking back at the ZEKE and 2D-LIF/DF spectra recorded via $5^{1}$ we see that there is still significant intensity in the ${ }^{+} 5^{1}$ and $5_{1}$ bands, respectively; indeed, the amount is consistent with the majority of the $5^{1} \mathrm{~m}^{0}$ intensity remaining. We thus conclude that, similar to the $6^{1} \mathrm{~m}^{1}$ case above, in the time-resolved experiments, ${ }^{35}$ rotational dephasing causes the loss of essentially all of the remaining ${ }^{+} 5^{1} m^{0} \mathrm{SEVI}$ signal, with a possible interacting level being $11^{1} 12^{1} \mathrm{~m}^{6(-)}$. In our ZEKE study of the $800 \mathrm{~cm}^{-1}$ bands ${ }^{23}$ we assigned a ZEKE band to ${ }^{+} 12^{1} m^{6-1}$, but reassigned this on the basis of our recently-reported 2D-LIF spectrum..$^{27} \mathrm{~A}$ large aspect of this reassignment was that we would expect the $12^{1} \mathrm{~m}^{6(-)}$ and $12^{1} 14^{1}$ levels to be close in wavenumber, in the same way that the $14^{2}$ and $14^{1} \mathrm{~m}^{6(-)}$ levels are close in wavenumber, ${ }^{21,22,26}$ as are $14^{1}$ and $m^{6(-)} .22$ Hence, here we would expect the $11^{1} 12^{1} 14^{1}$ and $11^{1} 12^{1} m^{6(-)}$ levels to be close together, with the $11^{1} 12^{1} 14^{1}$ level already having been deduced to be almost coincident with $5^{1}$ (subsection 3.3.2). What then of the remaining SEVI signal seen when exciting at $\sim 1230 \mathrm{~cm}^{-1}$ ? Again, we note that the resolution of the SEVI experiment is not sufficient to resolve all contributions, and so we suspect that there are underlying FC-active contributions to this SEVI signal that are independent of ${ }^{+} 5^{1}$, with ${ }^{+} 11^{3}$ and ${ }^{+} 11^{1} 12^{1} 14^{1}$ being possibilities at this wavenumber. Hence, we conclude that the residual signal of the decay of the " $+5^{1 "}$ SEVI signal ( $10 \%$ remaining) may be assigned to activity arising from uncoupled levels.

In summary, the $5^{1} \mathrm{SEVI}$ signal is lost by two mechanisms, with the ${ }^{+} 5^{1} m^{1}$ intensity being rapidly lost mainly via coupling to a bath of levels (dissipative IVR) - seen in both frequency- and time-resolved 
studies, while the ${ }^{+} 5^{1} m^{0}$ intensity is lost to rotational dephasing in the time-resolved experiments (the ${ }^{+} 5^{1} m^{1}$ signal could also be partially affected by rotational dephasing). The IVR causes the unstructured background in the SEVI, ZEKE and 2D-LIF/DF spectra, which arises from activity originating from a large number of bath states; the dephasing only occurs as a time-dependent interference effect, and consequently means that half the intensity is still present, as the ${ }^{+} 5^{1} m^{0}$ or $5_{1} m_{0}$ ZEKE or 2D-LIF/DF signals, respectively. The residual signal in the time-resolved SEVI spectrum when exciting via $5^{1}$ is concluded to arise from FC activity from overlapped, but uncoupled, $\mathrm{S}_{1}$ levels.

Consistent with comments above, we ruled out an assignment of the ZEKE band at $1606 \mathrm{~cm}^{-1}$, seen when exciting at $1208 \mathrm{~cm}^{-1}$, to ${ }^{+} 11^{1} 12^{1} \mathrm{~m}^{6(-)}$, since the excitation position is $25 \mathrm{~cm}^{-1}$ away from the $11^{1} 12^{1} 14^{1}$ transition, which has been deduced to be almost coincident with $5^{1}$ (subsection 3.3.2). Instead the $1606 \mathrm{~cm}^{-1}$ band has been assigned to ${ }^{+} 14^{1} 16^{1} 29^{1}$ on the basis that this fits the observed bands in the $S_{0}, S_{1}$ and $D_{0}{ }^{+}$states. Close to the $14^{1} 16^{1} 29^{1}$ band is the $16^{2}$ band (Figure 9), whose assignment is secure (despite the lack of a ZEKE spectrum) on the basis of the excellent agreement with the expected position of the $16_{2}$ band; furthermore, this is also in agreement with the observation of the $16^{2}$ band in $p D F B .48$ We can see a weak band that corresponds to $\left(14^{1} 16^{1} 29^{1}, 16_{2}\right)$, but do not see the partner $\left(16^{2}, 14_{1} 16_{1} 29_{1}\right)$ band, from which we conclude that it is unlikely these levels are in $\mathrm{FR}$, but that $16_{2}$ is $\mathrm{FC}$ active via $14^{1} 16^{1} 29^{1}$. We note that there is some $\mathrm{FC}$ activity of these when exciting $5^{1}$ and, most clearly, $6^{1}$.

Lastly, we discuss the excitation region close to $\sim 1250 \mathrm{~cm}^{-1}$ where the $12^{2}$ band is located. We have noted the observation of the $29_{1} m_{3(+)}$ band, and have associated this with $9^{1} 29^{1} \mathrm{~m}^{3(+)}$. We conclude this level is likely interacting with the $12^{2} \mathrm{~m}^{0}$ level, since $12^{2}$ appears to be the only bright level in this region (and is a transition also seen for $p D F B$ ). ${ }^{48}$ Although we do not see the $18_{1} m_{2}$ band, it is likely that there are some interactions with vibtor combinations involving this level, as we see evidence of weak emissions from $18^{2} 29^{1}$ and $11^{1} 18^{2}$ levels (not shown), and our earlier work ${ }^{24}$ has indicated interactions between $18^{2} \mathrm{~m}^{1}$ levels and levels such as $11^{1} 18^{1} \mathrm{~m}^{2}$. However, the interaction with the $29^{1} \mathrm{~m}^{3(+)}$ combination levels is the most likely explanation of the unstructured background that is present in the ZEKE (Figure 3) and 2D-LIF/DF (Figures 2 and 5) spectra when exciting at $1248 \mathrm{~cm}^{-1}$. In Figures 3 and 8 , we see that there are still substantial ${ }^{+} 12^{2}$ and $12_{2}$ ZEKE and 2D-LIF signals in the respective spectra, attributable to the $12^{2} m^{1}$ level.

In Table 2, we present the number of levels of different types that have the correct symmetry and lie close in wavenumber to the $6^{1}$ and $5^{1}$ levels, while in Figure 11 we show the rise in the number of $S_{1}$ 
vibrational and vibtor levels with increasing internal energy. The stark difference between the coupling of $6^{1}$ and $5^{1}$ cannot be straightforwardly related to the density of vibrational states since, as Table 2 and Figure 11 indicate, there are only a handful of extra totally-symmetric levels for the latter. There are more vibtor levels, with $~ 25 \%$ more levels that have the correct symmetry ( $a^{\prime}$ and $e^{\prime \prime}$ ) that could interact, but this is unlikely to explain the marked difference in behaviour between these two close-lying fundamentals. As such, and as discussed in Ref. 25, there is a certain amount of serendipity required, in that not only must there be levels of the correct symmetry to interact, but the coupling must also be efficient - with at least one doorway state.

We concluded in Ref. 25 that such coupling is aided by torsional motion, which allows out-of-plane (and so non-totally-symmetric) vibrations to become coupled to totally-symmetric vibrations, which will be FC-active. This idea was explored in Ref. 35 by Davies et al., building on the ideas of Moss et al. ${ }^{54}$, by modelling the van der Waals interaction between the methyl group and the closest phenyl hydrogens. It was found that, on the basis of the calculated vibrational motion, the $D_{5}$ vibration produced a larger interaction than did $D_{6}$. This is in apparent contrast to the localized motions, which are $\mathrm{C}-\mathrm{F}$ stretch for $D_{5}$ and $\mathrm{C}-\mathrm{CH}_{3}$ stretch for $D_{6}$, with the latter then being expected to cause more interaction. ${ }^{55,56}$ The explanation lies in the relative motions of the in-plane wags of the two closest phenyl $\mathrm{C}-\mathrm{H}$ bonds, and the $\mathrm{CH}_{3}$ stretch. Referring to Figure 11 , these may be seen to be in-phase in the case of $D_{6}$, so that the methyl group moves away from the phenyl ring, in sync with the two neighbouring $\mathrm{C}-\mathrm{H}$ bonds, and vice versa, so that the interaction between the sets of hydrogen atoms remains very similar; in contrast, since the $\mathrm{C}-\mathrm{CH}_{3}$ bond remains largely stationary in the $D_{5}$ motion (Figure 11), then the same $\mathrm{C}-\mathrm{H}$ wagging motions cause a greater interaction than in $D_{6}$. This shows that there is vibrational mode specificity in determining the efficiency of IVR, as concluded by Davies et al. ${ }^{35}$ The analysis here emphasises that the whole vibrational motion should be considered when examining this, not just looking at localized motions.

Also, Figure 11 shows that the $D_{18}$ mode has out-of-plane "wagging" of the two C-H bonds closest to the methyl group, suggestive of their being able to "waft" the methyl group (see also Ref. 24). Also, the motion of $D_{29}$ indicates significant methyl motion, causing it to come into closer contact with a phenyl $\mathrm{C}-\mathrm{H}$ bond, causing interaction. Hence, both vibrations may reasonably be expected to cause motion of the methyl group. 


\section{Concluding Remarks}

In this work, we have presented further explicit evidence that vibration-torsion interactions are a key factor in facilitating IVR in molecules that contain a methyl group. Further, we have shown that there is a certain amount of serendipity involved, particularly at moderately low internal energy, in doorway states being present close to a ZOB state and which can enable efficient coupling to the background bath states, whose density is increasing rapidly with increasing internal energy. The efficiency of a doorway state in effecting IVR is dependent on having the correct symmetry, being energetically close to the ZOB state, and being well-coupled both to the ZOB state and a significant number of the bath states - this is likely to be linked to its motion and how this interacts with other motions in the molecule, particularly the methyl group. In this regard, we have suggested that the whole vibrational motion should be considered, and not simply local motions.

The above conclusions have been obtained from both fluorescence and ZEKE spectroscopies, with consistent activity in both producing the most reliable results. The 2D-LIF spectra, in particular, have aided in the identification of overlapped activity and particularly allowing the extraction of the vibtor activity. We have been further aided by earlier analyse ${ }^{26,27}$ of the lower wavenumber regions that underpin much of the energy level structure in the present wavenumber region. The detailed knowledge of the wavenumbers of the vibrational and vibtor levels in the $S_{0}, S_{1}$ and $D_{0}{ }^{+}$states (present work, Ref. 23 and work cited therein) provides great insight into the couplings that occur. Notably, the greater detail of the present study has allowed further insight into the results of time-resolved studies.

It is clear that many of these ideas will be more generally applicable, with coupling between chromophore-localized vibrations and wide-amplitude motions that will provide routes for IVR in a range of molecules, with one key set being biomolecules which often contain $\mathrm{NH}_{2}$ and $\mathrm{CH}_{3}$ groups, as well as other flexible side chains. 


\section{Acknowledgements}

We are grateful to the EPSRC for funding (grant EP/L021366/1). The EPSRC and the University of Nottingham are thanked for studentships to D.J.K. and W.D.T. We are grateful to Lewis Warner who helped to record some 2D-LIF images as part of an Undergraduate Summer Bursary project funded by The Royal Society of Chemistry. We are grateful for discussions with Warren Lawrance and Jason Gascooke (Flinders, Adelaide), and Katharine Reid (Nottingham). 
Table 1: Correspondence of the $C_{2 v}$ point group symmetry classes with those of the $G_{12}$ molecular symmetry group. Also indicated are the symmetries of the $D_{i}$ vibrations and the different pure torsional levels. ${ }^{a}$

\begin{tabular}{|l|l|l|l|}
\hline$C_{2 v}$ & $G_{12}$ & $D_{i}{ }^{\mathrm{b}}$ & $m$ \\
\hline$a_{1}$ & $a_{1}{ }^{\prime}$ & $D_{1}-D_{11}$ & $0,6(+)$ \\
\hline$a_{2}$ & $a_{2}{ }^{\prime}$ & $D_{12}-D_{14}$ & $6(-)$ \\
\hline$b_{1}$ & $a_{2}{ }^{\prime \prime}$ & $D_{15}-D_{20}$ & $3(-)$ \\
\hline$b_{2}$ & $a_{1}{ }^{\prime \prime}$ & $D_{20}-D_{30}$ & $3(+)$ \\
\hline & $e^{\prime}$ & & 2,4 \\
\hline & $e^{\prime \prime}$ & & 1,5 \\
\hline
\end{tabular}

a Symmetries of vibtor levels can be obtained by combining the vibrational symmetry (in $G_{12}$ ) with those of the pure torsional level, using the $D_{3 h}$ point group direct product table.

${ }^{\mathrm{b}}$ The $D_{i}$ labels are described in Ref. 41, where the vibration mode diagrams can also be found. 
Table 2: Numbers of vibrations and vibtor levels that can interact with $D_{6}$ or $D_{5}{ }^{\mathrm{a}}$

\begin{tabular}{|l|c|c|}
\hline Vibrational subset & $D_{6}$ & $D_{5}$ \\
\hline Totally symmetric vibrations & 20 & 22 \\
\hline All vibrations & 49 & 92 \\
\hline$A_{1}{ }^{\prime}$ Vibtors & & 77 \\
\hline$E^{\prime \prime}$ Vibtors $^{\mathrm{c}}$ & 67 & 132 \\
\hline$A_{1}{ }^{\prime}+E^{\prime \prime}$ vibtors & 102 & 209 \\
\hline All vibtors & 169 & 585 \\
\hline
\end{tabular}

a Numbers of $S_{1}$ levels calculated within a $\pm 20 \mathrm{~cm}^{-1}$ window of each fundamental, based upon calculated harmonic vibrational wavenumbers ${ }^{23}$ and approximate energies of the torsional levels. It is clear that both anharmonicity and vibration-torsional coupling will produce minor changes in these numbers, but are not expected to be a large.

${ }^{\mathrm{b}}$ Symmetry-allowed coupling to $m=0$ levels of totally-symmetric vibrations.

c Symmetry-allowed coupling to $m=1$ levels of totally-symmetric vibrations. 


\section{Figure Captions}

Figure 1: Overview of the $0-1250 \mathrm{~cm}^{-1}$ region of the REMPI spectrum of the $S_{1} \leftarrow \mathrm{S}_{0}$ transition in $p$ FT. See text for further comments.

Figure 2: The 1190-1250 $\mathrm{cm}^{-1}$ region of the $\mathrm{S}_{1} \leftarrow \mathrm{S}_{0}$ transition in $p \mathrm{FT}$. (i) recorded using REMPI spectroscopy; (ii) using laser-induced fluorescence; and (iii) by vertical integration of the 2D-LIF spectrum. See text for further comment.

Figure 3: ZEKE spectra of $p F T$ recorded across the $\sim 1200 \mathrm{~cm}^{-1}$ excitation wavenumber region. At the top of the figure a REMPI spectrum of the relevant region is presented, with the letters indicating the positions which were used for recording ZEKE spectra. The ZEKE traces are indicated with the same lettering, except for that of the origin (top ZEKE trace), which is shown for comparative purposes. Some key assignments are indicated, with further discussion presented in the text.

Figure 4: Composite 2D-LIF spectrum including all regions recorded as part of the present work. Further discussion and expansion of various regions are found elsewhere in the paper. The main excitations are indicated at the top of the spectrum.

Figure 5: Dispersed fluorescence spectra recorded via (i) $0^{0}$; (ii) $6^{1}$; (iii) $5^{1}$; and (iv) $12^{2}$. Of note is the almost completely flat baseline for (i), a small amount of rising background with widespread activity to high wavenumber in (ii), and a significant amount of rising background with widespread activity in (iii) and (iv).

Figure 6: Slices of the 2D-LIF spectrum showing the key activity following excitation of $6^{1}$ and $5^{1}$. Selected assignments are given, with a number of these discussed in the text.

Figure 7: Indications of the expected $\mathrm{S}_{1} \leftarrow \mathrm{S}_{0}$ activity arising from combinations of features at $\sim 400$ $\mathrm{cm}^{-1}$ and $\sim 800 \mathrm{~cm}^{-1}$. The former region of the spectrum is shown offset by amounts corresponding to the three main bands of the latter region. The approximate positions of the various vibrational combination bands is given, although not all are observed; further movement of levels as a result of interactions is expected to occur - see text for further discussion.

Figure 8: The top of the figure shows the 2D-LIF features considered, while the bottom traces show integrated activity of the emission of some key 2D-LIF features activity across the excitation region indicated. Integration was carried out over a range of emission wavenumbers that included the main activity, whilst avoiding overlap with neighbouring features.

Figure 9: Region of the 2D-LIF spectrum that shows various $\Delta v=0$ bands (boxed) that show activity in this region. A couple of bands arising from Franck-Condon activity following $6^{1}$ excitation are shown for orientation purposes with Figures 4 and 6 , and to allow judgement of the relative intensities.

Figure 10: Vibtor activity seen in the 2D-LIF spectra. The top image shows the $\Delta(v, m)=02 \mathrm{D}$-LIF bands of two of the main vibtor levels. The bottom traces show portions of the three dispersed fluorescence spectra where the $\Delta(v, m)=0$ and/or associated activity is most clearly seen. Note that 
since the associated vibtor levels are acting as doorway states, their $\Delta(v, m)=0$ activity is low, being spread through the coupled levels. See text for further discussion.

Figure 11: In the upper part of the figure, the number of states within $10 \mathrm{~cm}^{-1}$ windows is plotted as a function of the $S_{1}$ state internal energy. The positions of $6^{1}$ and $5^{1}$ are indicated. Below this, the mode diagrams of the $D_{6}$ and $D_{5}$ fundamentals are shown, as are those for $D_{18}$ and $D_{29}$. In the latter two cases, it can be seen how the motion can cause the vibrations to interact with the torsional motion of the methyl group. 
Figure 1

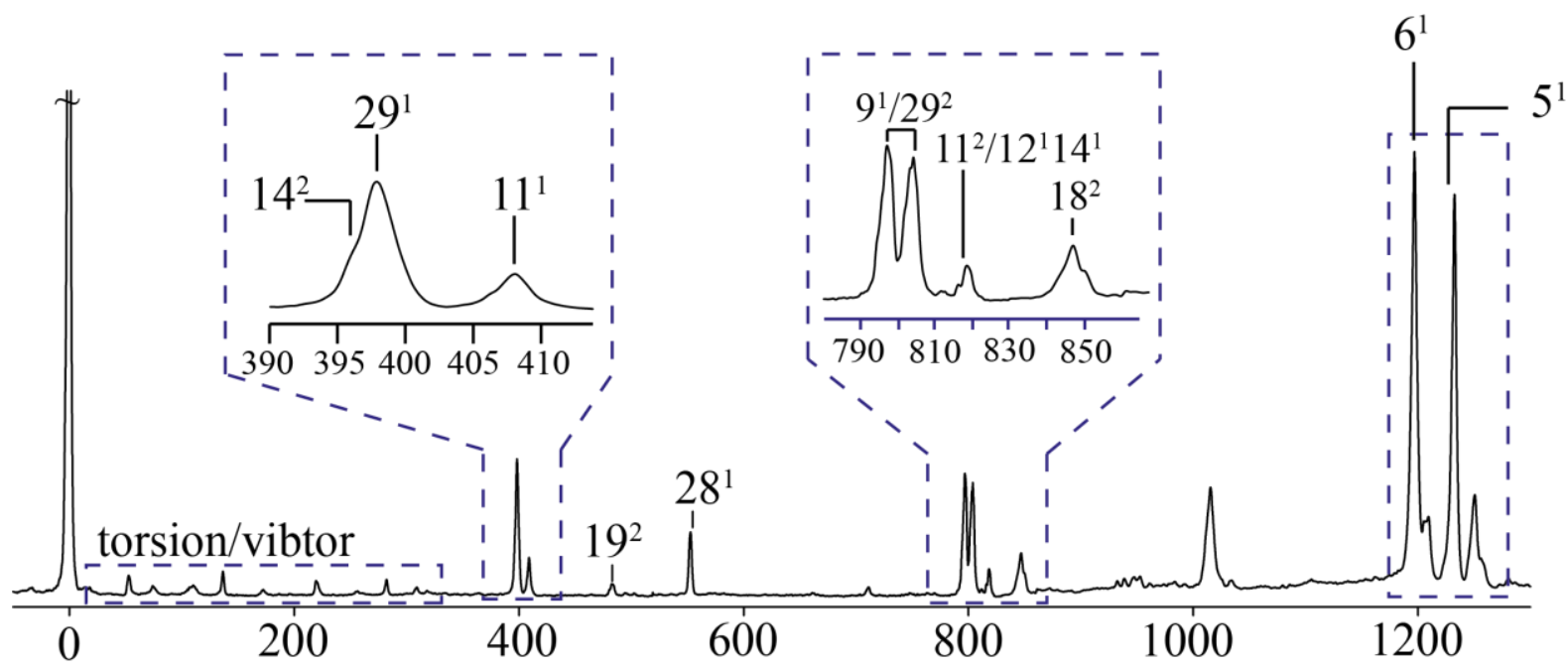

Wavenumber relative to $\mathrm{S}_{1}$ origin $/ \mathrm{cm}^{-1}$ 
Figure 2
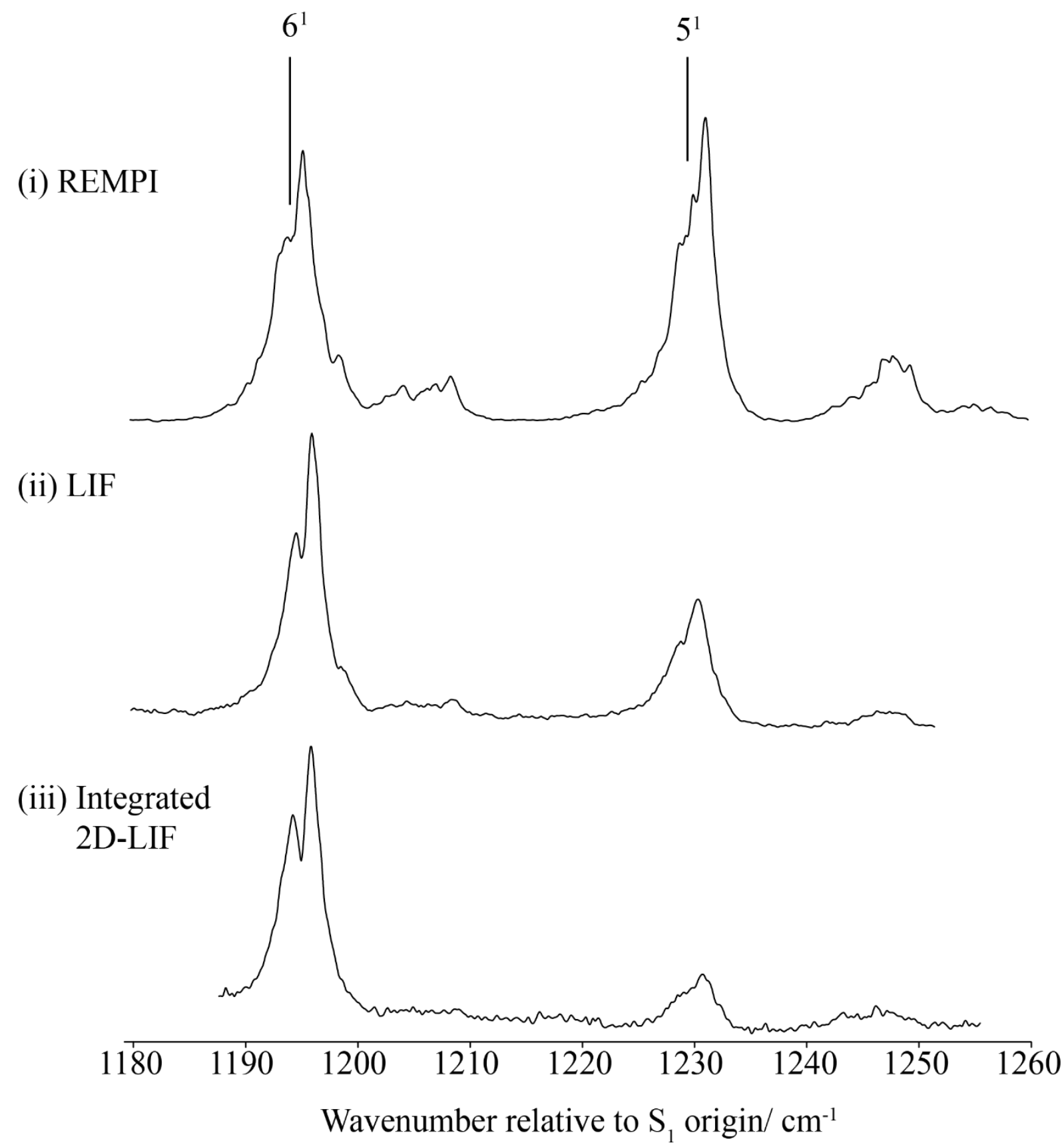
Figure 3

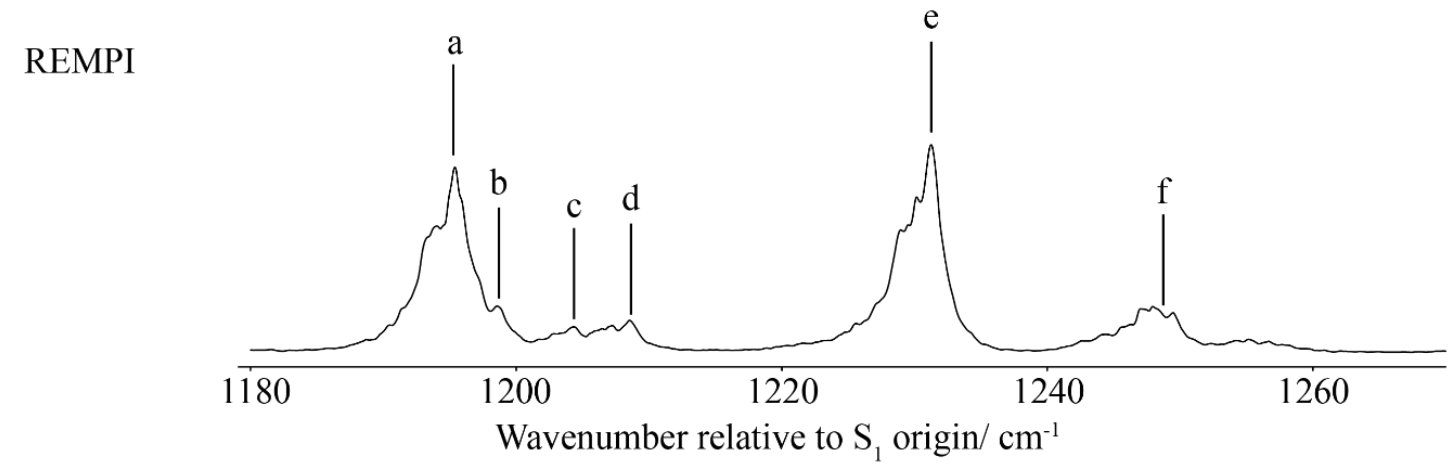

ZEKE

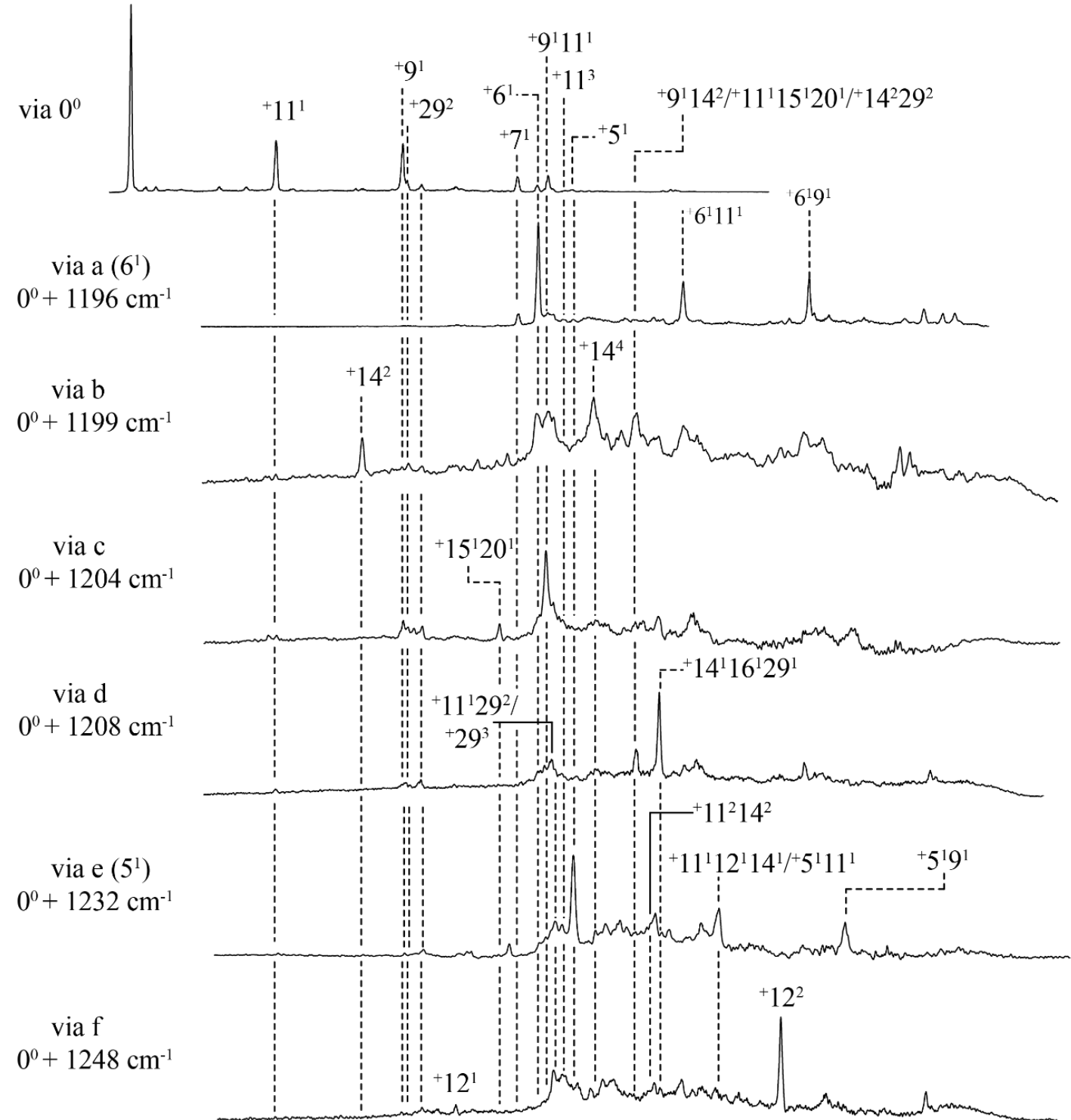

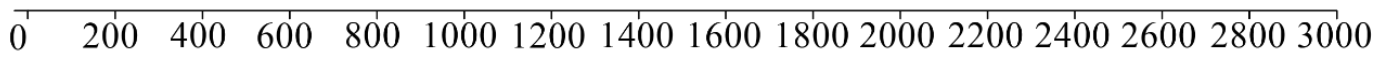
Wavenumber relative to $\mathrm{D}_{0}^{+}$origin $/ \mathrm{cm}^{-1}$ 
Figure 4

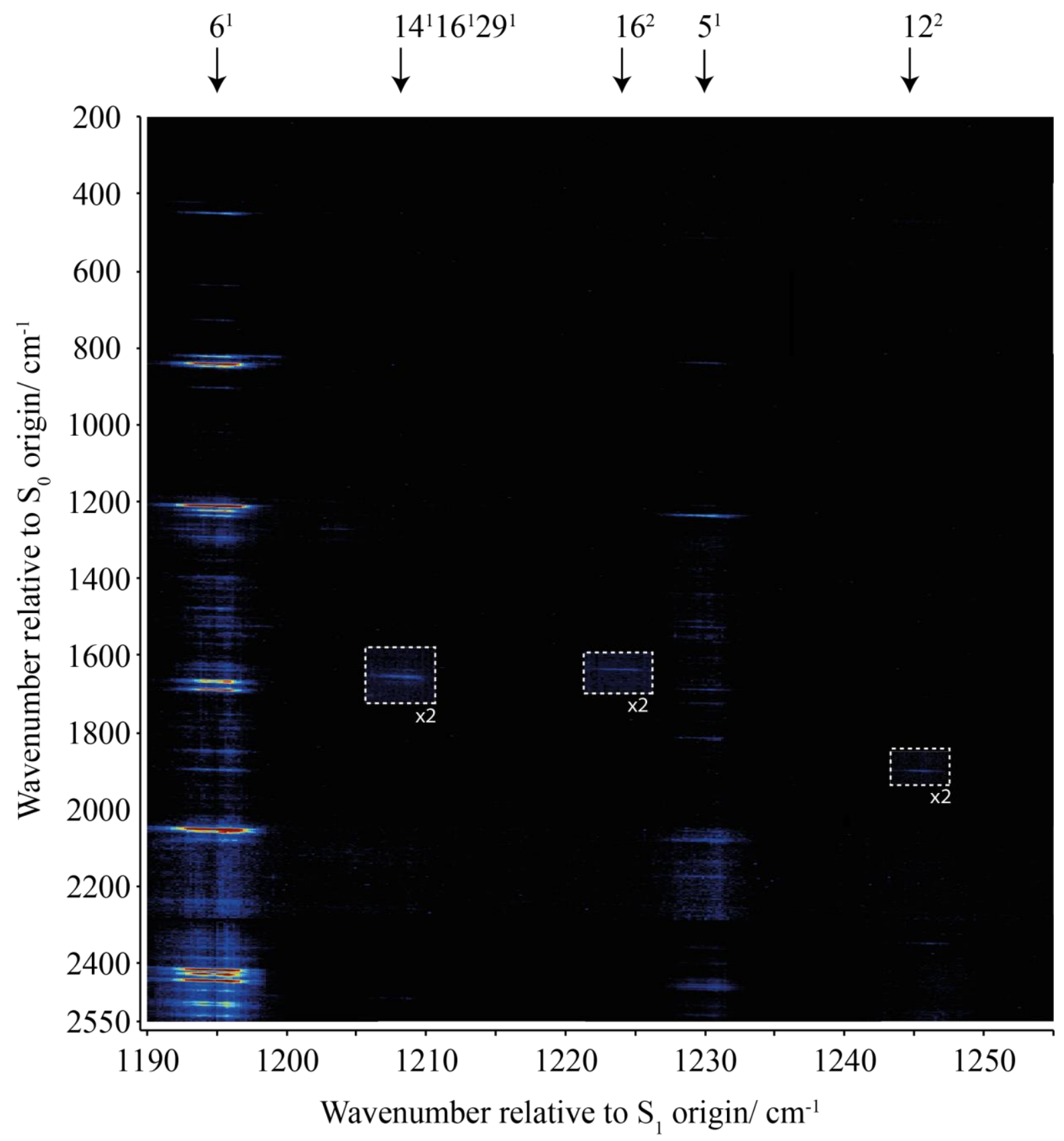


Figure 5

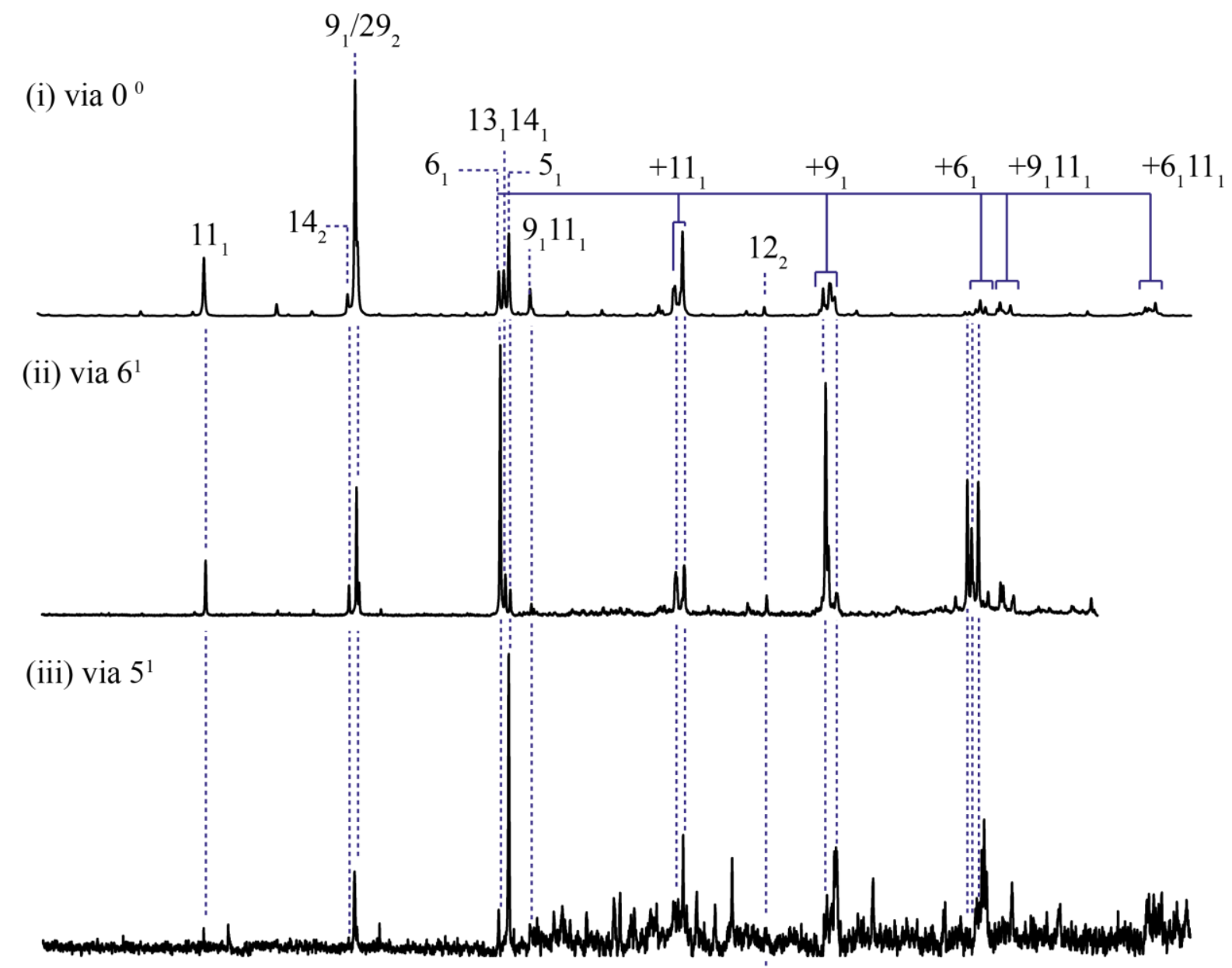

(iv) via $12^{2}$

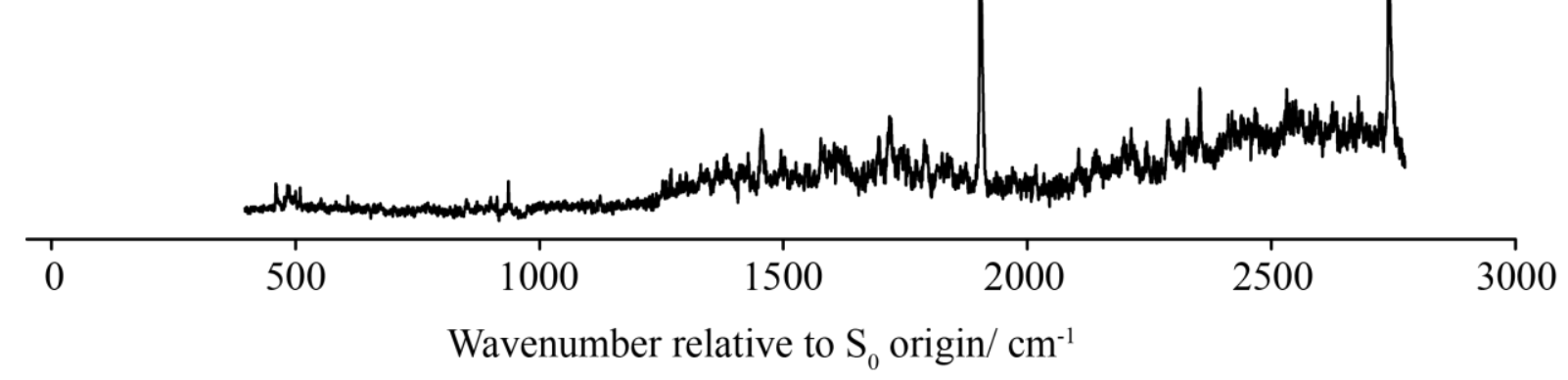


Figure 6

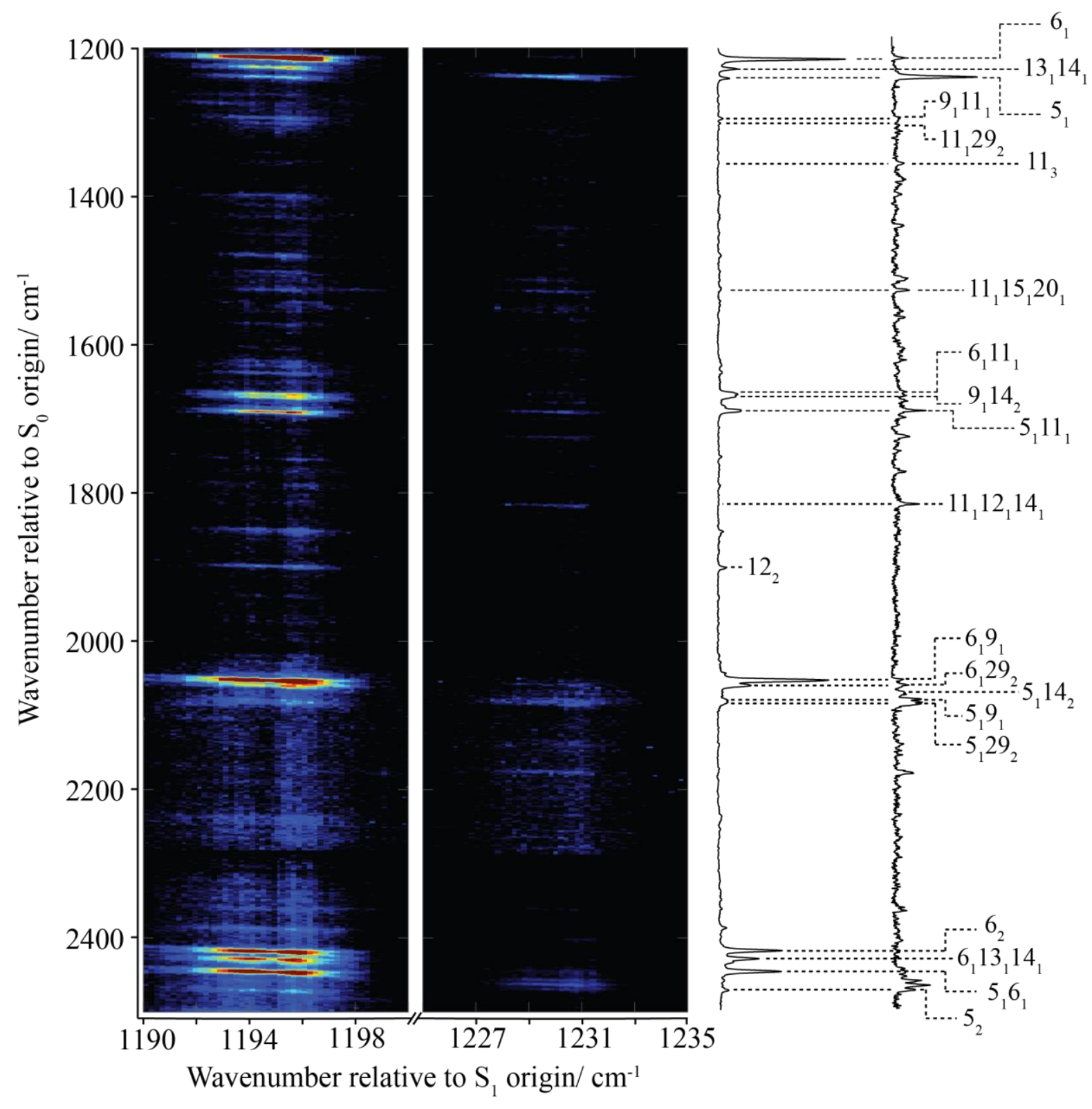


Figure 7

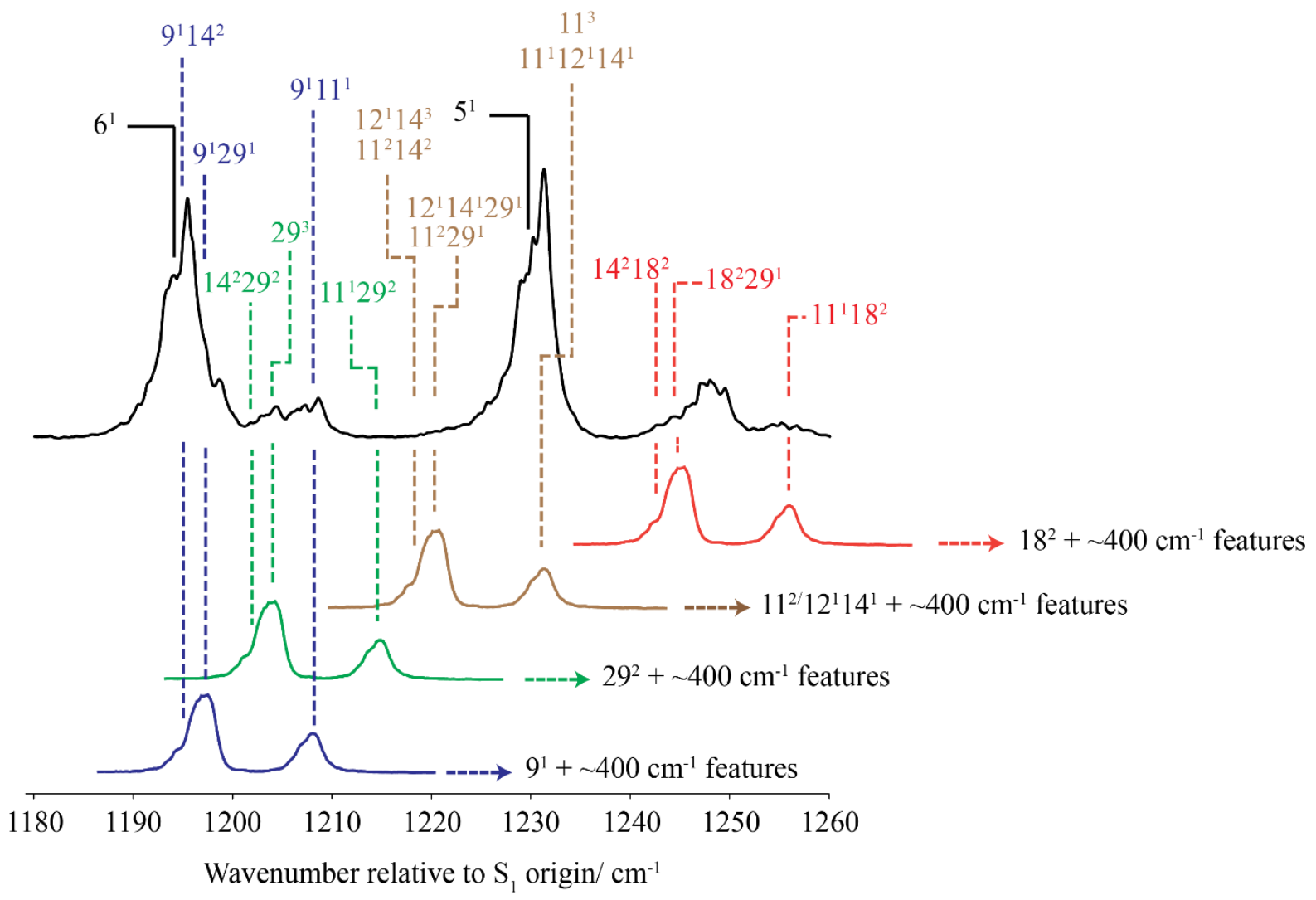


Figure 8

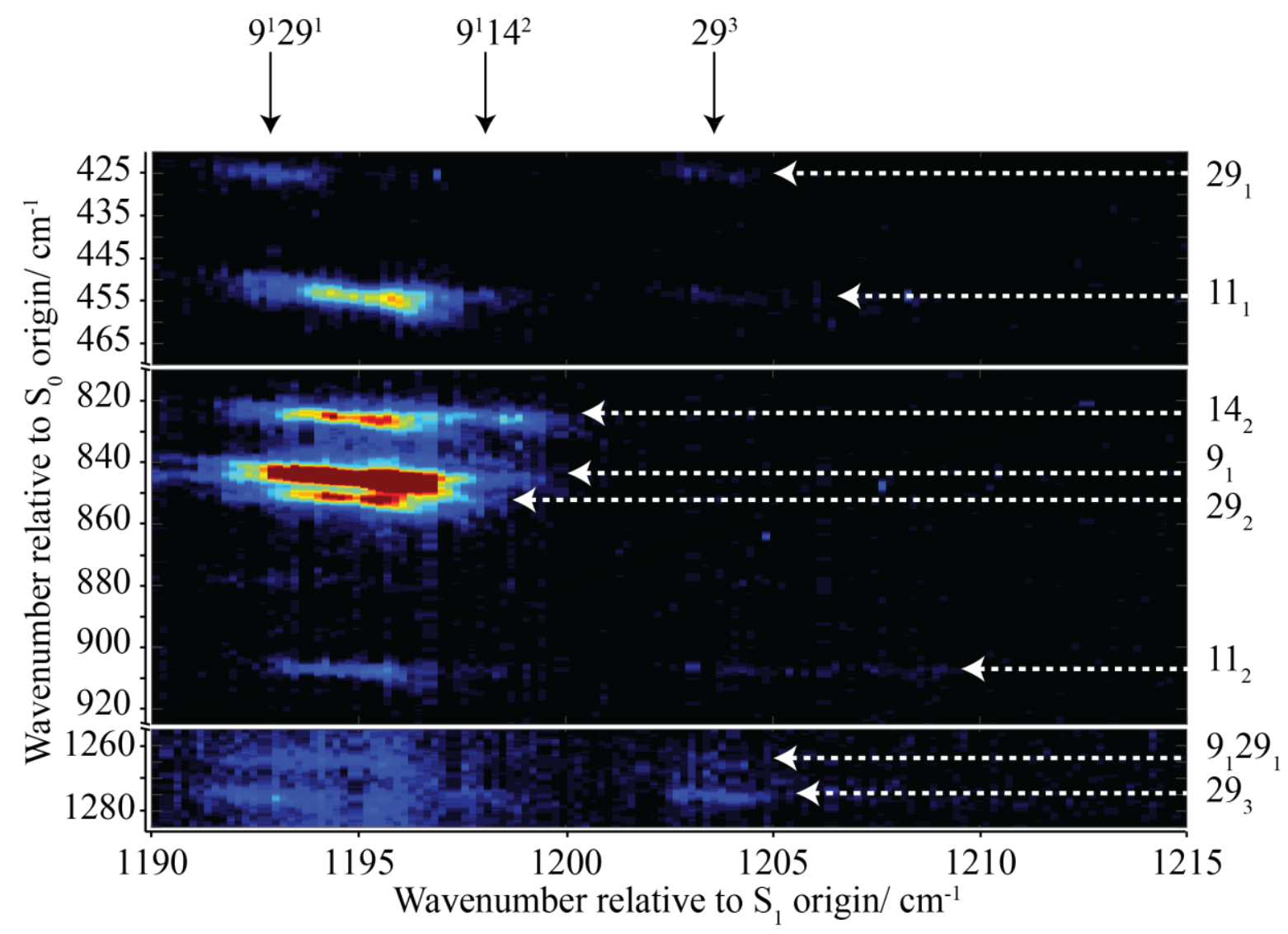

via 29
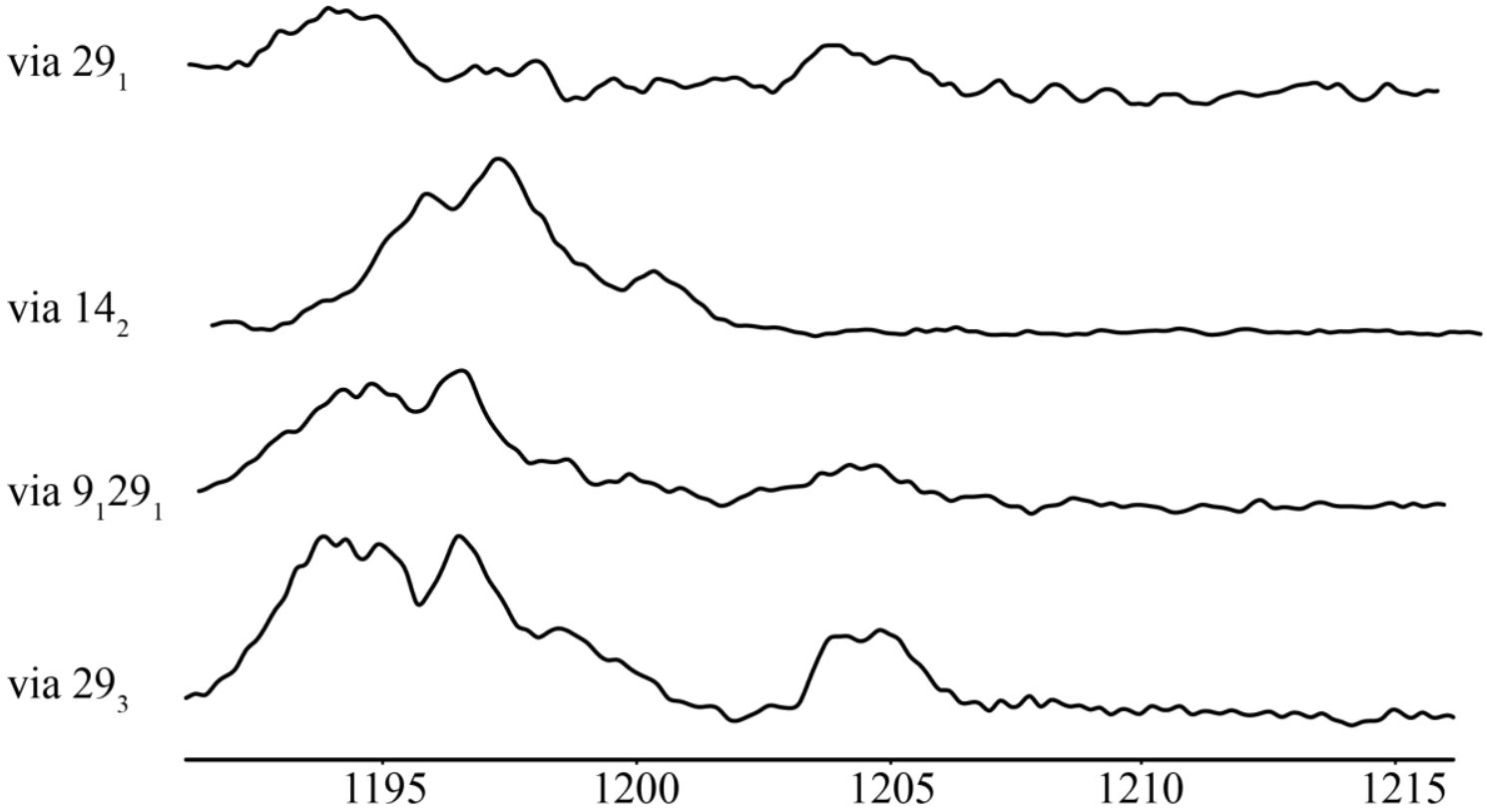

Wavenumber relative to $S_{1}$ origin $/ \mathrm{cm}^{-1}$ 


\section{Figure 9}

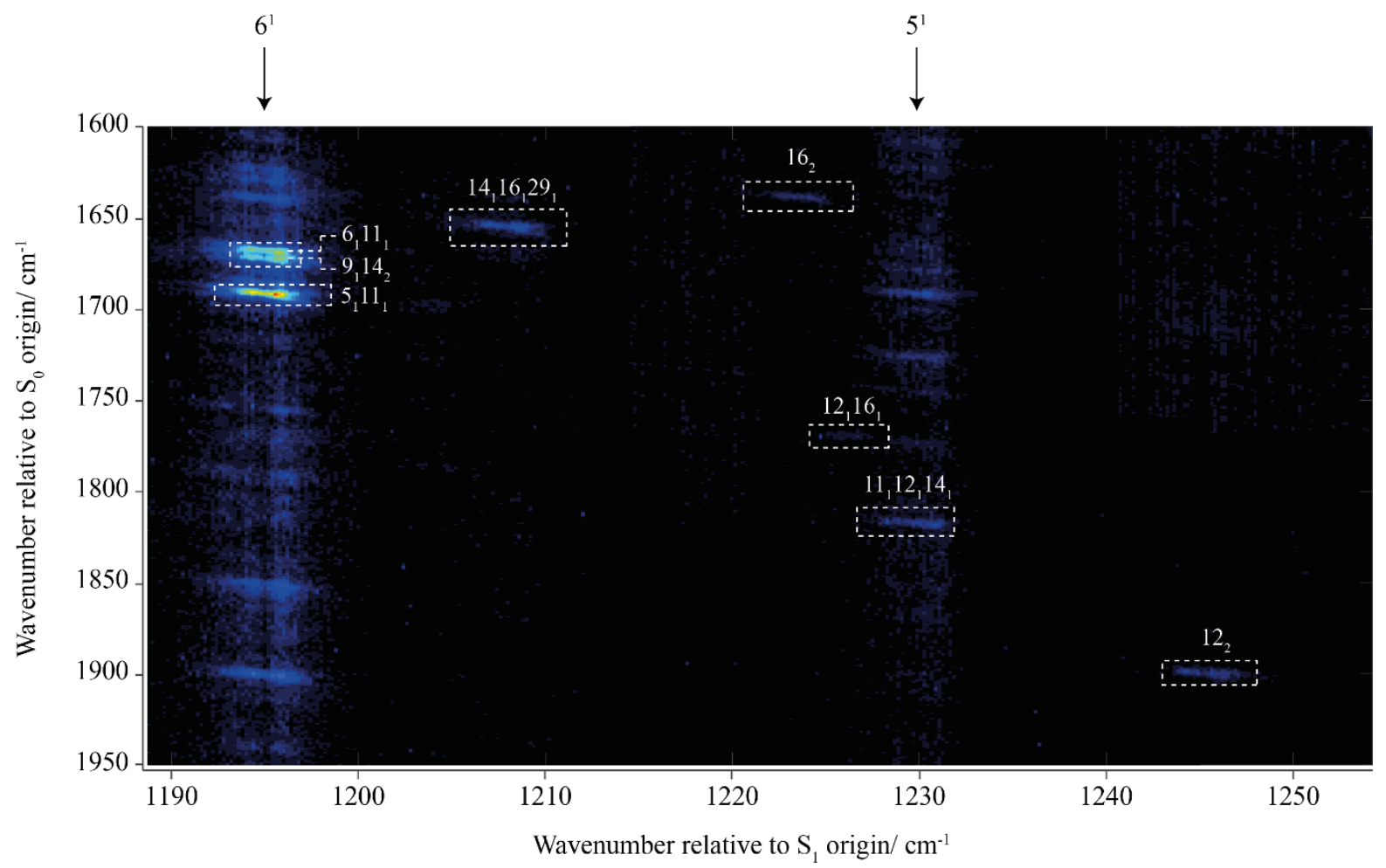


Figure 10
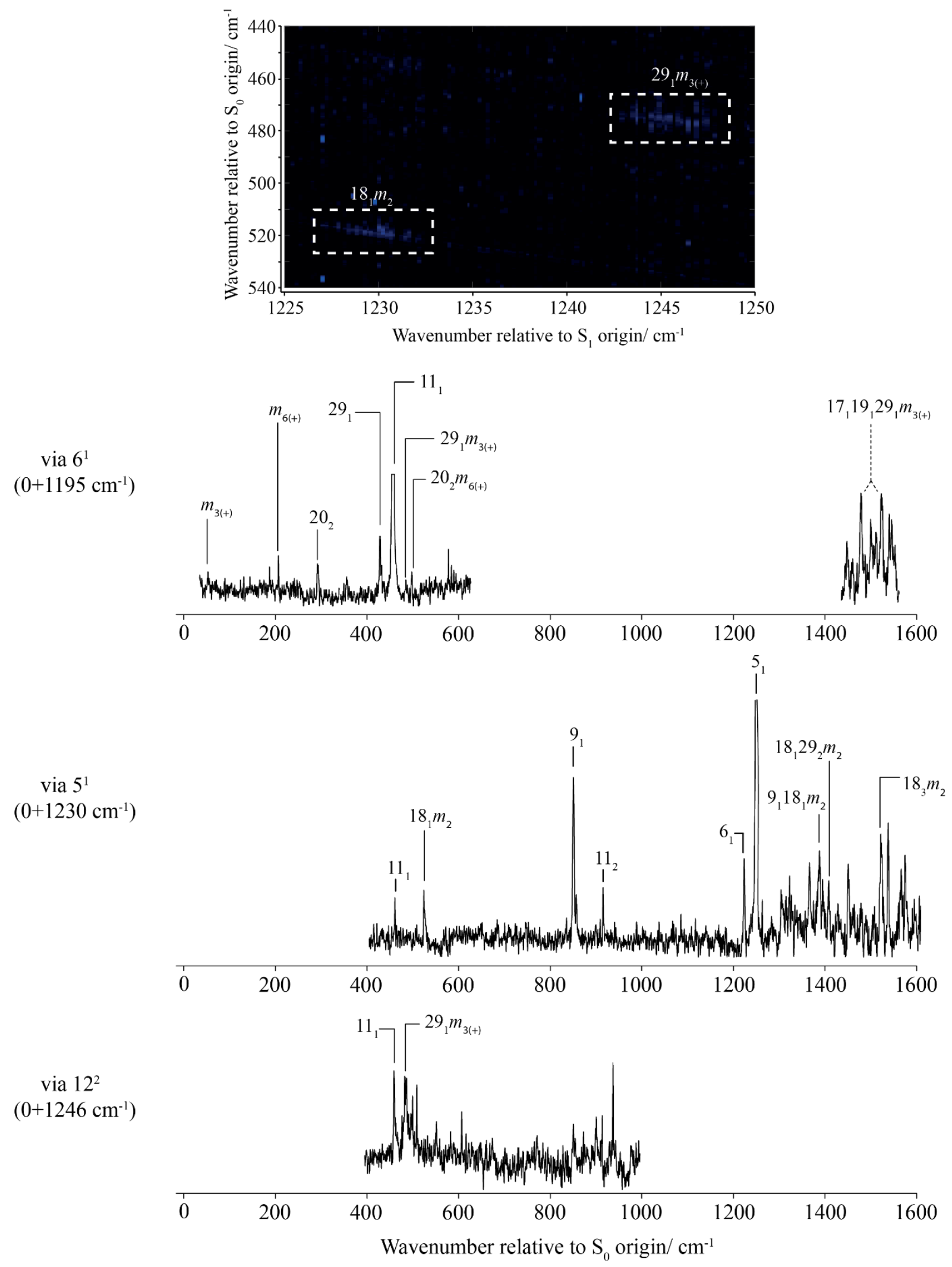
Figure 11

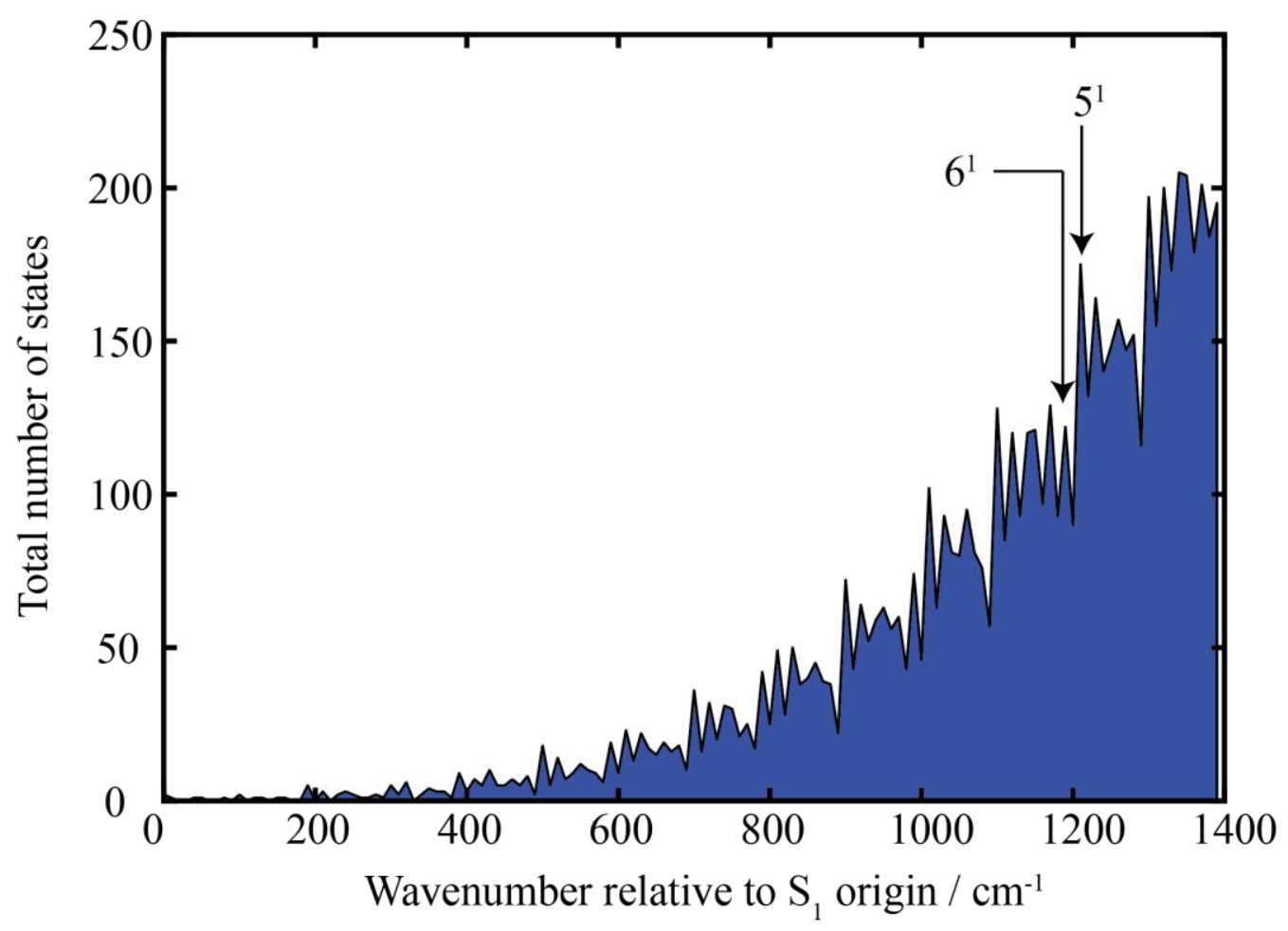

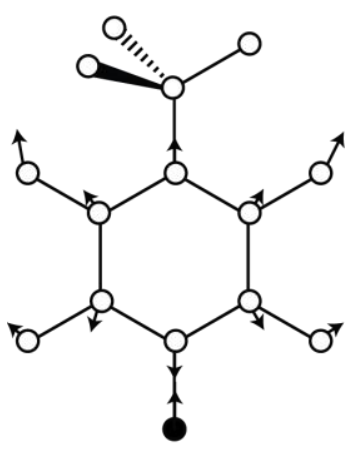

$D_{5}\left(a_{1}\right)$

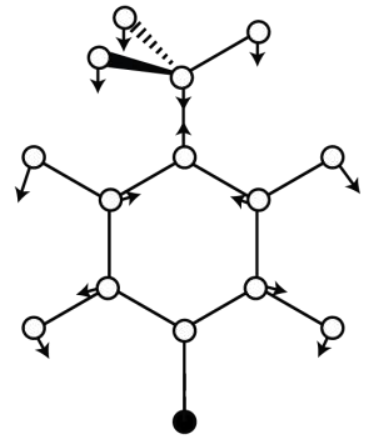

$D_{6}\left(a_{1}\right)$

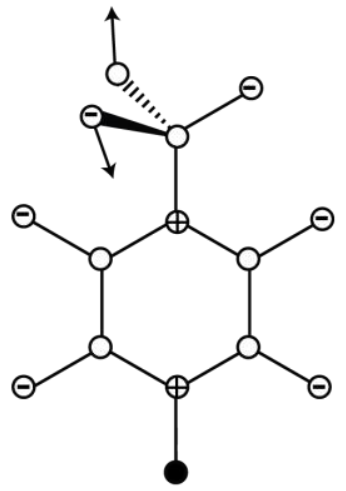

$D_{18}\left(b_{1}\right)$

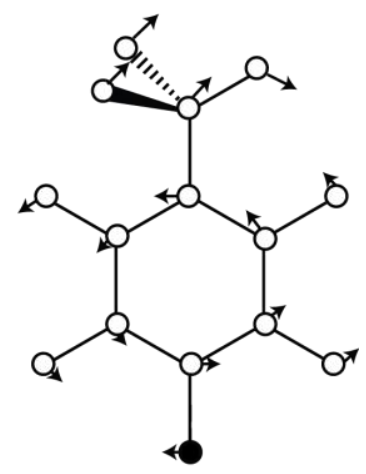

$D_{29}\left(b_{2}\right)$ 


\section{References}

${ }^{1}$ C. S. Parmenter and B. M. Stone, J. Chem. Phys. 84, 4710 (1986).

${ }^{2}$ D. B. Moss and C. S. Parmenter, J. Chem. Phys. 98, 6897 (1993).

${ }^{3}$ Z.-Q. Zhao and C. S. Parmenter, Mode Selective Chemistry (Kluwer, 1991) Eds. J. Jortner, R. D.

Levine, and B. Pullman. Jerusalem Symp. Quant. Chem. Biochem. 24, 127 (1991).

${ }^{4}$ Z.-Q. Zhao, C. S. Parmenter, D. B. Moss, A. J. Bradley, A. E. W. Knight, and K. G. Owens, J. Chem. Phys. 96, 6362 (1992).

${ }^{5}$ Z.-Q. Zhao, PhD Thesis, Indiana University (1992).

${ }^{6}$ P. J. Timbers, C. S. Parmenter, and D. B Moss, J. Chem. Phys. 100, 1028 (1994).

${ }^{7}$ Q. Ju, C. S. Parmenter, T. A. Stone, and Z.-Q. Zhao, Isr. J. Chem. 37, 379 (1997).

${ }^{8}$ R. A. Walker, E. C. Richard, K.-T. Lu, and J. C. Weisshaar, J. Phys. Chem. 99, 12422 (1995).

${ }^{9}$ E. C. Richard, R. A. Walker, and J. C. Weisshaar, J. Chem. Phys. 104, 4451 (1996).

${ }^{10}$ S. H. Feldus, M. J. Schroeder, R. A. Walker, W.-K. Woo, and J. C. Weisshaar, Int. J. Mass Spectrom. Ion Proc. 159, 231 (1996).

${ }^{11}$ J. A. Davies, A. M. Green, A. M. Gardner, C. D. Withers, T. G. Wright, and K. L. Reid, Phys. Chem. Chem. Phys. 16, 430 (2014).

${ }^{12}$ A. M. Gardner, A. M. Green, V. M. Tamé-Reyes, K. L. Reid, J. A. Davies, V. H. K. Parkes, and T. G. Wright, J. Chem. Phys. 140, 114308 (2014).

${ }^{13}$ A. M. Gardner, A. M. Green, V. M. Tamé-Reyes, V. H. K. Wilton, and T. G. Wright, J. Chem. Phys. 138,134303 (2013).

${ }^{14}$ E. A. Virgo, J. R. Gascooke, and W. D. Lawrance, J. Chem. Phys. 140, 154310 (2014).

${ }^{15}$ J. R. Gascooke, E. A. Virgo, and W. D. Lawrance, J. Chem. Phys. 142, 024315 (2015).

${ }^{16}$ J. R. Gascooke, E. A. Virgo, and W. D. Lawrance, J. Chem. Phys. 143, 044313 (2015).

17 J. R. Gascooke and W. D. Lawrance, J. Molec. Spec., 318, 53 (2015).

${ }^{18}$ V. L. Ayles, C. J. Hammond, D. E. Bergeron, O. J. Richards, and T. G. Wright, J. Chem. Phys. 126, 244304 (2007).

${ }^{19}$ C. J. Hammond, V. L. Ayles, D. E. Bergeron, K. L. Reid, and T. G. Wright, J. Chem. Phys. 125, 124308 (2006).

20 J. A. Davies, A. M. Green, A. M. Gardner, C. D. Withers, and T. G. Wright, Phys. Chem. Chem. Phys. 16,430 (2014).

${ }^{21}$ A. M. Gardner, W. D. Tuttle, L. Whalley, A. Claydon, J. H. Carter, and T. G. Wright, J. Chem. Phys. 145, 124307 (2016). 
22 J. R. Gascooke, L. D. Stuart, P. G. Sibley, and W. D. Lawrance, J. Chem. Phys. 149, 074301 (2018). This work contains considerable extra information in its supplementary information. Note, however, that the excitation wavenumber axis is incorrect in Figure S9.

${ }^{23}$ W. D. Tuttle, A. M. Gardner, L. E. Whalley, and T. G. Wright, J. Chem. Phys. 146, 244310 (2017).

${ }^{24}$ A. M. Gardner, W. D. Tuttle, L. E. Whalley, and T. G. Wright, Chem. Sci. 9, 2270 (2018).

${ }^{25}$ W. D. Tuttle, A. M. Gardner, L. E. Whalley, D. J. Kemp, and T. G. Wright, Phys. Chem. Chem. Phys. 21, 14133 (2019).

${ }^{26}$ D. J. Kemp, A. M. Gardner, W. D. Tuttle, and T. G. Wright, Molec. Phys. (2019) In press. https://doi.org/10.1080/00268976.2018.1554865

${ }^{27}$ D. J. Kemp, L. E. Whalley, A. M. Gardner, W. D. Tuttle, L. G. Warner, and T. G. Wright, J. Chem. Phys. 150, 064306 (2019).

${ }^{28}$ A. M. Gardner, W. D. Tuttle, P. Groner, and T. G. Wright, J. Chem. Phys. 146, 124308 (2017).

${ }^{29}$ W. D. Tuttle, A. M. Gardner, K. B. O'Regan, W. Malewicz, and T. G. Wright, J. Chem. Phys. 146, 124309 (2017).

${ }^{30}$ J. R. Gascooke and W. D. Lawrance, Eur. Phys. J. D 71, 287 (2017).

${ }^{31}$ W. T. Cave and H. W. Thompson, Faraday Soc. Trans. 9, 35 (1950).

${ }^{32}$ T. Cvitaš and J. M. Hollas, Molec. Phys., 20, 645 (1971).

${ }^{33}$ C. J. Seliskar, M. A. Leugers, M. Heaven, and J. L. Hardwick, J. Molec. Spect. 106, 330 (1984).

${ }^{34}$ K. Okuyama, N. Mikami, and M. Ito, J. Phys. Chem. 89, 5617 (1985).

35 J. A. Davies, L. E. Whalley, and K. L. Reid, Phys. Chem. Chem. Phys. 19, 5051 (2017).

${ }^{36}$ E. B. Wilson, Jr, Phys. Rev., 45, 706 (1934).

${ }^{37}$ G. Varsányi, Assignments of the Vibrational Spectra of Seven Hundred Benzene Derivatives, Wiley, New York, 1974.

${ }^{38}$ R. S. Mulliken, J. Chem. Phys., 23, 1997 (1955).

${ }^{39}$ G. Herzberg, Molecular Spectra and Molecular Structure II. Infrared and Raman Spectra of Polyatomic Molecules, Krieger, Malabar, 1991.

${ }^{40}$ A. M. Gardner and T. G. Wright, J. Chem. Phys., 135, 114305 (2011).

${ }^{41}$ A. Andrejeva, A. M. Gardner, W. D. Tuttle, and T. G. Wright, J. Molec. Spect. 321, 28 (2016).

${ }^{42}$ P. J. Breen, J. A. Warren, E. R. Bernstein, and J. I. Seeman, J. Chem. Phys. 87, 1917 (1987).

${ }^{43}$ E. Fermi, Z. Phys., 71, 250 (1931).

${ }^{44}$ J. R. Gascooke and W. D. Lawrance, J. Chem. Phys. 138, 134302 (2013).

${ }^{45}$ N. T. Whetton and W. D. Lawrance, J. Phys. Chem. 93, 5377 (1989).

${ }^{46}$ Z.-Q. Zhao and C. S. Parmenter, Ber. Bunsenges. Phys. Chem. 99, 536 (1995).

${ }^{47}$ J. A. Davies and K. L. Reid, Phys. Rev. Lett., 109, 193004 (2012). 
${ }^{48}$ D. J. Kemp, A. M. Gardner, W. D. Tuttle, J. Midgley, K. L. Reid, and T. G. Wright, J. Chem. Phys. 149, 094301 (2018).

${ }^{49}$ W. D. Tuttle, A. M. Gardner, and T. G. Wright, Chem. Phys. Lett. 684, 339 (2017).

${ }^{50}$ D. J. Kemp, L. E. Whalley, W. D. Tuttle, A. M. Gardner, B. T. Speake, and T. G. Wright, Phys. Chem. Chem. Phys. 20, 12503 (2018).

${ }^{51}$ G. A. Bethardy, X. Wang, and D. S. Perry, Can. J. Chem., 72, 652 (1994).

${ }^{52}$ D. S. Perry, G. A. Bethardy, and X. Wang, Ber. Bunsenges. Phys. Chem. 99, 530 (1995).

53 P. M. Felker and A. H. Zewail, J. Chem. Phys. 82, 2294 (1985).

${ }^{54}$ D. B. Moss, C. S. Parmenter, and G. E. Ewing, J. Chem. Phys. 86, 51 (1987).

${ }^{55}$ G. A. Bethardy, X. Wang, and D. S. Perry, Can. J. Chem. 72, 652 (1994).

${ }^{56}$ D. S. Perry, G. A. Bethardy, and X. Wang, Ber. Bunsenges. Phys. Chem. 99, 530 (1995). 